\title{
SKP2 inactivation suppresses prostate tumorigenesis by mediating JARID1B ubiquitination
}

\author{
Wenfu Lu ${ }^{1}$, Shenji Liu ${ }^{1}$, Bo Li ${ }^{1}$, Yingqiu Xie $^{1}$, Christine Adhiambo $^{1}$, Qing Yang ${ }^{1}$, \\ Billy R. Ballard ${ }^{2}$, Keiichi I. Nakayama ${ }^{3}$, Robert J. Matusik ${ }^{4}$, Zhenbang Chen ${ }^{1}$ \\ ${ }^{1}$ Department of Biochemistry and Cancer Biology, Meharry Medical College, TN 37208, USA \\ ${ }^{2}$ Department of Pathology, Anatomy and Cell Biology, Meharry Medical College, TN 37208, USA \\ ${ }^{3}$ Department of Molecular and Cellular Biology, Medical Institute of Bioregulation, Kyushu University, Fukuoka 812-8582, \\ Japan \\ ${ }^{4}$ Department of Urologic Surgery, Vanderbilt University School of Medicine, TN 37232, USA \\ Correspondence to: \\ Zhenbang Chen, e-mail: zchen@mmc.edu \\ Keywords: PTEN, SKP2, JARIDIB, TRAF6, histone modifications, prostate cancer \\ Received: September 02, $2014 \quad$ Accepted: November 11, $2014 \quad$ Published: December 23, 2014
}

\section{ABSTRACT}

\begin{abstract}
Aberrant elevation of JARID1B and histone H3 lysine 4 trimethylation (H3K4me3) is frequently observed in many diseases including prostate cancer (PCa), yet the mechanisms on the regulation of JARID1B and H3K4me3 through epigenetic alterations still remain poorly understood. Here we report that Skp2 modulates JARID1B and H3K4me3 levels in vitro in cultured cells and in vivo in mouse models. We demonstrated that Skp2 inactivation decreased $\mathrm{H} 3 \mathrm{~K} 4 \mathrm{me} 3$ levels, along with a reduction of cell growth, cell migration and malignant transformation of Pten/Trp53 double null MEFs, and further restrained prostate tumorigenesis of Pten/Trp53 mutant mice. Mechanistically, Skp2 decreased the K63-linked ubiquitination of JARID1B by E3 ubiquitin ligase TRAF6, thus decreasing JARID1B demethylase activity and in turn increasing H3K4me3. In agreement, Skp2 deficiency resulted in an increase of JARID1B ubiquitination and in turn a reduction of $\mathrm{H} 3 \mathrm{~K} 4 \mathrm{me} 3$, and induced senescence through JARID1B accumulation in nucleoli of PCa cells and prostate tumors of mice. Furthermore, we showed that the elevations of Skp2 and H3K4me3 contributed to castration-resistant prostate cancer (CRPC) in mice, and were positively correlated in human PCa specimens. Taken together, our findings reveal a novel network of SKP2- JARID1B, and targeting SKP2 and JARID1B may be a potential strategy for PCa control.
\end{abstract}

\section{INTRODUCTION}

Prostate cancer $(\mathrm{PCa})$ is the second leading cause of cancer-related deaths in American males [1]. Molecular mechanisms leading to this malignancy are complicate but need to be elucidated in order to develop efficient pathways-targeted chemotherapies. SKP2 (S-phase kinase associated protein-2) is an E3 ligase and an F-box protein component of SKP2 SCF complex (Skp1-Cul1F-Box) to trigger the ubiquitin-mediated degradation of p27 and other proteins $[2,3]$. As a proto-oncogene, SKP2 overexpression is frequently observed in various human cancers including PCa $[4,5]$, and aberrant elevation of SKP2 is associated with poor prognosis of cancers $[6,7]$.
Studies demonstrated that SKP2 has oncogenic impact on the initiation and progression of $\mathrm{PCa}$, which correlating with castration resistant prostate cancer (CRPC). Emerging evidence revealed that SKP2 plays an essential role in cell cycle proliferation, cellular senescence, cancer progression, and metastasis $[6,8-10]$. This phenomenon is likely a result of degrading the downstream substrates in cell cycles such as $\mathrm{p} 27[8,11]$. SKP2 regulates a variety of downstream targets hence alters a broad spectrum of signaling cascades [11]. However, SKP2 affects the expression of proteins other than its substrates such as ATF4 [8], RhoA and Miz1 [10] and C-Myc [12, 13], suggesting its essential roles independent of E3 ligase in PCa. Recent studies indicated that SKP2 has novel 
functions in glycolysis and tumorigenesis through the regulation of AKT ubiquitination in human breast cancer [14]. Moreover, SKP2 is reported to affect the levels of androgen receptor via ubiquitination in $\mathrm{PCa}$ [15]. Despite these advancements, the role of SKP2 on the regulation of histone modifications in human PCa still remains elusive.

Global histone modifications including lysine methylation and acetylation have been reported to correlate with human cancers [16-18]. Histone methylation is a dynamic biochemical process that is tightly controlled by histone methyltransferases and histone demethylases to balance biological functions in cells. Deregulation of these epigenetic modification enzymes contributes to the development and progression of $\mathrm{PCa}$ through the activation or suppression of gene functions [17-20]. Recent reports show that the increase of JARID1B/ KDM5B/PLU1, a specific histone demethylase for H3K4 trimethylation and dimethylation, is frequently observed in $\mathrm{PCa}$ specimens, implicating its potential oncogenic roles in PCa [17-20]. The deregulation of trimethylation of lysine 4 on histone $\mathrm{H} 3$ ( $\mathrm{H} 3 \mathrm{~K} 4 \mathrm{me} 3$ ), is contributing to the development of primary $\mathrm{PCa}$ as well as progression to CRPC [18]. In addition, aberrant elevation of $\mathrm{H} 3 \mathrm{~K} 4 \mathrm{me} 3$ also contributes to an epigenetic switch of a number of oncogenes and tumor suppressor genes in PCa cells and furthermore to cancer stem cells $[19,21]$. However, the mechanisms underlying the deregulation of $\mathrm{H} 3 \mathrm{~K} 4 \mathrm{me} 3$ by JARID1B and the relevance with SKP2 in cancers are poorly understood.

Substantial evidence indicates SKP2 plays a critical role in tumorigenesis by regulating a variety of downstream effectors of cellular progresses [6, 8-11, 14]. For example, SKP2 is reported to contribute to the epigenetic silencing of DAB2IP in PCa [22]. Although the role of histone methyltransferase MLL in PCa is unclear, it was reported that leukemogenic MLL fusions were resistant to SKP2-mediated degradation, suggesting the involvement of SKP2 in epigenetic modification mechanisms of histone in Leukemia [23]. Among various histone modifications, $\mathrm{H} 3 \mathrm{~K} 4 \mathrm{me} 3$ is significantly increased in both localized and hormone refractory PCa $[17,24]$. Therefore, we reasoned that SKP2 may be involved in the epigenetic events including histone methylation modifications by regulating histone methylation/ demethylation enzymes in PCa.

In this study, we discovered that the aberrant levels of $\mathrm{H} 3 \mathrm{~K} 4 \mathrm{me} 3$ are remarkably reduced by SKP2 ablation in PCa cells and tumors. We demonstrated that SKP2 determines $\mathrm{H} 3 \mathrm{~K} 4 \mathrm{me} 3$ levels through inhibiting the K63linked ubiquitination of JARID1B by E3 ubiquitin ligase TRAF6, resulting in repression of JARID1B demethylase activity. Our findings on histone modifications highlight the implications of a combinatorial targeting on SKP2 and JARID1B for PCa treatment.

\section{RESULTS}

\section{Skp2 deficiency restrains the prostate tumor growth of Pten/Trp53 mutant mice}

To explore the role of SKP2 on epigenetics and the relevance on $\mathrm{PCa}$ progression in vivo, we wished to investigate whether Skp2 deficiency suppresses prostate tumorigenesis through affecting the functional coupling of JARID1B and H3K4me3 in mouse models. We focused on JARID1B as it controls H3K4me 3 and its abnormal upregulation is frequently observed in $\mathrm{PCa}$ [17-20]. To test this possibility, we took advantage of Pten/Trp53 mouse model to generate Pten/Trp53/Skp2 conditional triple null $\left(\right.$ Pten $^{p c-/} ;$ Trp $53^{p c-/} ;$ Skp2 $\left.2^{-/}\right)$mutant mice, and subsequently assessed their prostate tumorigenesis. In agreement with previous report [25], Pten/Trp53 conditional double null $\left(\right.$ Pten $^{p c-1} ;$ Trp $\left.3^{p c--}\right)$ mice developed prostate tumors at 3 months of age, and the average weight of anterior prostates (AP) was 5-fold heavier than that of the age-matched wild type (WT) cohort (Supplementary Figure S1A and $\mathrm{S} 1 \mathrm{~B})$. The enlargement of AP in Pten $^{p c-/} ; \operatorname{Trp}^{53^{p c-/}}$ mice was noticeable when dissected, and marked pathological changes including high-grade prostatic intraepithelial neoplasia (HG-PIN) and invasive cancer were observed in all mice (Supplementary Figure S1C). Importantly, Skp2 deficiency resulted in a suppression of development of prostate tumorigenesis in $\mathrm{Pten}^{p c-1}$; Trp $53^{p c-1}$ mice, while Skp2 null alone did not cause morphological changes of prostates. The average AP weight of $\mathrm{Pten}^{p c-/}$; Trp53 $53^{p-1-}$; $S k p 2^{-/}$mice reduced about $50 \%$ as compared to that of Pten $^{p c-1} ; \operatorname{Trp}^{53^{p c-/}}$ mice at 3 months of age $(P<0.05$, Supplementary Figure S1A and S1B). Prostate tumors in Pten $^{p c-1}$; Trp 53 $3^{p c-/}$ mice developed microinvasion with cells in atypical nucleus, while age-matched $\operatorname{Pten}^{p c-1}$; $\operatorname{Trp53} 3^{p--/}$; Skp $2^{-/-}$mice showed the less severe abnormality of prostate glands with PIN lesions (Supplementary Figure $\mathrm{S} 1 \mathrm{C})$. These data indicate that Skp2 deficiency reduces the development and progression of $\mathrm{PCa}$ in vivo.

Since Pten/Trp53 double null mice died of enlarged prostate tumors by 5-6 months of age, we then assessed the sustained impact of Skp2 deficiency on prostate tumorigenesis of Pten/Trp 53 mutant mice. Remarkably, Skp2 deficiency significantly suppressed the growth of prostate tumors of $\mathrm{Pten}^{p c-1}$; Trp $53^{p c-1}$ mice (Supplementary Figure S1D). The average tumor mass of Pten ${ }^{p--1}$; Trp 53 $3^{p--1}$; Skp2 $2^{-/}$mice reduced 2.68-fold as compared to that of Pten ${ }^{p--1}$; Trp53 $3^{p c-/}$ mice (Figure 1A, $P<0.001, N=12$ mice). Pathological analysis revealed that prostate tumors of $\mathrm{Pten}^{p c-1}$; Trp $53^{p c-1}$ mice developed poorly differentiated cancer (sarcomatoid) without discernible structures of prostate glands (Figure 1B). In contrast, prostate tumors of $\operatorname{Pten}^{p c-/}$; Trp53 $3^{p c-1}$; Skp2 $2^{-/-}$ mice were much smaller with visible forms of glandular 
A

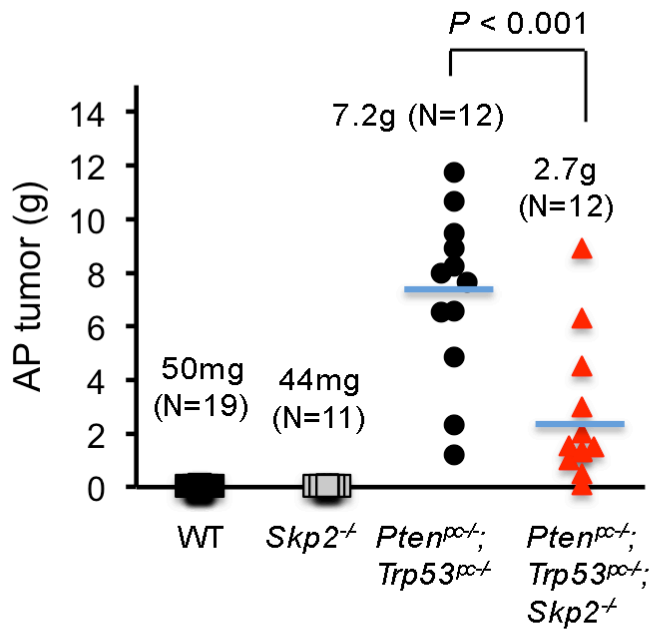

C

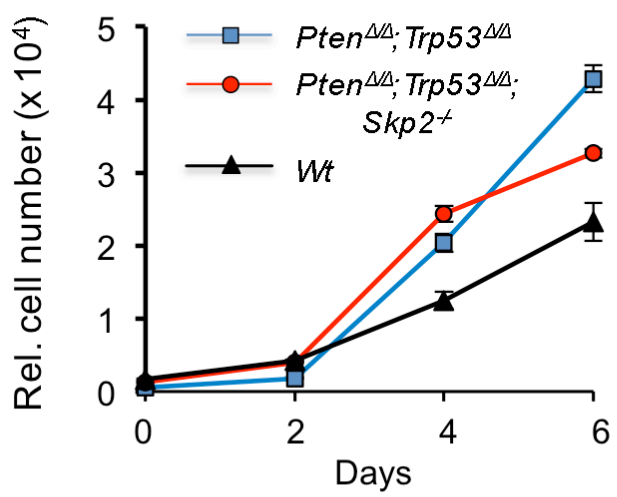

E

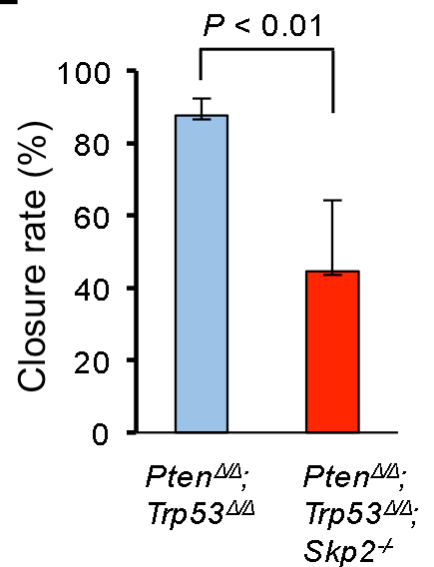

B
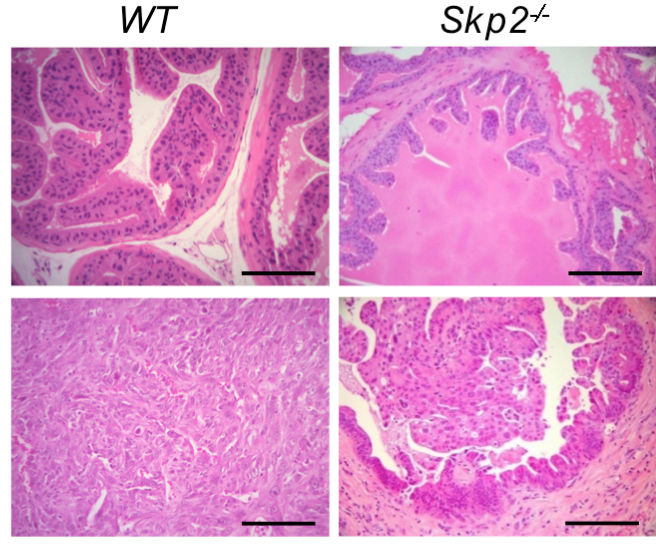

$\operatorname{Pten}^{p c-1-}$ Trp53 $3^{\text {pc- }-}$

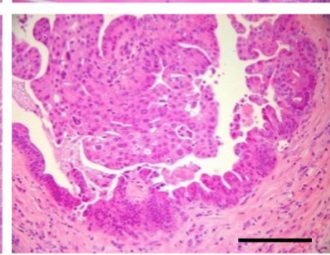

$\operatorname{Pten}^{p c-\alpha} ; \operatorname{Trp} 53^{p c-\alpha}$ Skp2-1-

D

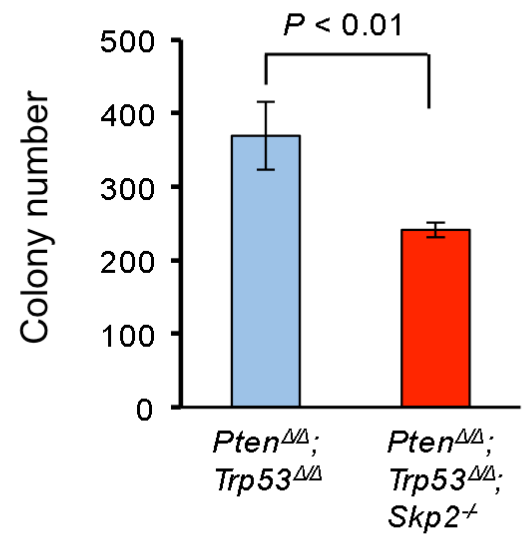

Figure 1: Skp2 inactivation suppresses prostate cancer progression in vivo in mice and cell growth of MEF by regulating JARID1B and H3K4me3 in vitro. (A) Quantification analysis of average weights of anterior prostate (AP) tumors of mice at 5-6 months of age. The number of mice for each group is as indicated. The representative biopsies of AP from indicated genotypes of mice are shown in Supplementary Figure S1D. (B) H\&E staining of prostate tissues from indicated genotypes of mice at 5-6 months of age. Scale bars represent $100 \mu \mathrm{m}$. (C) Effects of Skp2 inactivation on the cell proliferation of Pten/Trp53 null MEFs. (D) Effects of Skp2 inactivation on soft agar transformation of Pten/Trp53 null MEFs. (E) Wound healing (migration) assay of Pten/Trp53 null MEFs upon SKP2 inactivation (Also see Supplementary Figure S1E). (F) Left panel, Western blot analysis of protein levels of JARID1B and H3K4me3 upon Skp2 inactivation in Pten/Trp53 null MEFs. Right panel, quantification analysis of protein levels for JARID1B and H3K4me3 in MEFs upon Skp2 inactivation. Error bars for C-F represent means \pm SD. 
structures as HGPIN, and few cells displayed atypical nucleus. These results demonstrated that Skp2 inactivation restrains prostate cancer progression of Pten/Trp 53 mutant mice.

\section{Skp2 ablation reduces H3K4me3 levels through JARID1B to inhibit cell migration in MEFs}

To delineate the impact of Skp2 in the regulation of H3K4me3, we first chose to examine the effect of Skp2 inactivation on the expression of H3K4me3 in Pten/Trp53 double null MEFs in vitro, and in Pten ${ }^{p c-1}$; Trp $53^{p c-1}$ mice in vivo. By following the same strategy reported previously $[25,26]$, we prepared Pten/Trp53 $\left(\right.$ Pten $\left.^{1 / 4} ; \operatorname{Trp} 53^{4 / 4}\right)$ double null and Pten/Trp53/Skp2 triple null (Pten ${ }^{\Delta / 4}$; Trp53 $3^{\Delta / \Delta}$; $\left.S k p 2^{--}\right)$MEFs, and investigated the biological effects of Skp2 deficiency in Pten/Trp53 double null MEFs. In agreement with previous reports, a combined loss of Pten and Trp53 genes in MEFs led to a significant increase of cell proliferation as compared to WT MEFs. Remarkably, the cell proliferation of Pten/Trp53/Skp2 triple null MEFs was significantly reduced as compared to Pten/Trp53 double null MEFs (Figure 1C). As Pten/Trp53 double null MEFs showed the soft agar transformation, we further assessed the suppressive effect of Skp2 inactivation on this malignant feature. Our results showed that Skp2 inactivation resulted in a significant reduction in colony size and numbers (Figure 1D, $P<0.01$ ). In addition, Skp2 ablation resulted in a significant reduction of cell migration (the closure rate) (Figure 1E, $P<0.01$, Supplementary Figure S1E).

We next evaluated H3K4me3 levels in Pten/Trp53 double null and Pten/Trp53/Skp2 triple null MEFs. Consistent with previous reports $[7,8]$, Skp2 deficiency resulted in an increased level of p27 protein in Pten/ Trp53 double null MEFs (Data not shown). Importantly, Skp2 deficiency resulted in a significant reduction of H3K4me3 levels (3-fold), suggesting a pivotal role of Skp2 in the regulation of H3K4 trimethylation, at least in Pten and Trp53 double null background (Figure 1F). Meanwhile, Skp2 loss alone did not result in any reduction of H3K4me3 levels when compared to that in WT MEFs (Data not shown). Our results suggest that aberrant elevation of H3K $4 \mathrm{me} 3$ levels by oncogenic insults may be a Skp2-dependent cascade. To investigate the mechanisms on the regulation of $\mathrm{H} 3 \mathrm{~K} 4 \mathrm{me} 3$ by Skp2, we examined the effects of Skp2 ablation on the protein levels of JARID1B, a specific histone demethylase of H3K $4 \mathrm{me} 3 / 2$ that is frequently overexpressed in PCa [17-20]. Western results revealed that JARID1B levels were aberrantly elevated upon the concomitant inactivation of both Pten and Trp53 genes as compared to WT (Data not shown). Remarkably, Skp2 inactivation led to a striking elevation of JARID1B levels in Pten/Trp53 MEFs, and protein levels of JARID1B in Pten/Trp53/Skp2 triple null MEFs increased 2-fold as compared to that in Pten/Trp53 double null MEFs (Figure 1F, right panel), companying with a 3-fold decrease of H4K4me3 level. These data indeed provided biological evidence on a functional relationship between JARID1B and H3K4me3 in cells under defined oncogenic insults. Furthermore, our results revealed a novel function of Skp2 on the regulation of histone modification enzyme JARID1B to determine the levels of H3K 4 trimethylation in cells. Taken together, these results demonstrated that Skp2 inactivation resulted in a reduction of $\mathrm{H} 3 \mathrm{~K} 4 \mathrm{me} 3$, which may contribute to the inhibition of cell proliferation, transformation, and migration in MEFs.

\section{Skp2 deficiency decreases H3K4me3 levels in prostate tumors of Pten/Trp53 mutant mice}

We then investigated whether the striking suppression of prostate tumorigenesis by Skp2 deficiency in Pten $^{p c-/}$; Trp $53^{p c-/}$; Skp $2^{--}$mice was caused by an alteration of the H3K4me3/ JARID1B coupling. Consistent with the findings in vitro, we discovered a reverse correlation between H3K4me3 and JARID1B levels in mouse prostate tumors in vivo (Figure 2A). Quantification analysis revealed that Skp2 inactivation indeed led to about 2-fold increase of JARID1B and consequently a marked 2-fold decrease of H3K4me3 levels in Pten $^{p c-1}$; Trp $53^{p c-1} ;$ Skp $2^{-/}$mice, as compared to Pten ${ }^{p c-1}$; Trp $53^{p c-/}$ mice (Figure 2B). Importantly, IHC staining results of consecutive sections revealed that the lesions with elevated accumulation of JARID1B in nucleus showed decreased levels of H3K4me3 (Figure 2A), indicating Skp2 may regulate JARID1B in $\mathrm{PCa}$ cells. These lines of evidence in vitro and in vivo support that Skp2 is an essential oncogenic factor for $\mathrm{PCa}$ progression by driving the dysregulation of $\mathrm{H} 3 \mathrm{~K} 4$ trimethylation through JARID1B.

\section{H3K4me3 correlates with the recurrent growth of prostate tumors in Pten/Trp53 mice}

Aberrations of histone methylation and acetylation are associated with clinical outcome in several cancers [27, 28]. Elevated expression of $\mathrm{H} 3 \mathrm{~K} 4 \mathrm{me} 3$ is frequently found in CRPC as compared to benign tumor specimens [17], suggesting the dysregulation of $\mathrm{H} 3 \mathrm{~K} 4$ trimethylation may contribute to castration resistance. Therefore, we wished to obtain new insights into the dysregulation of $\mathrm{H} 3 \mathrm{~K} 4 \mathrm{me} 3$ on CRPC using mouse models. To do so, we generated luciferase-labeled Pten/Trp53 conditional double null $\left(\right.$ Luc $^{p c+} ;$ Pten $^{p c-1} ; \operatorname{Trp} 53^{p c-1}$ referred to Luc/Pten/Trp53) mutant mice, in which the expression of firefly luciferase gene is turned on concomitantly with the inactivation of Pten and Trp53 genes in prostatic epithelium driven by Probasin-Cre 4 after puberty [25, 29]. As shown in the biopsy and the bioluminescence imaging (BLI), the luciferase positivity was confined to prostate tumors of mutant mice (Supplementary Figure S2). Therefore, we used Luc/Pten/Trp53 mutant mice to recapitulate features of human CRPC by performing castration at 14 weeks of 
A
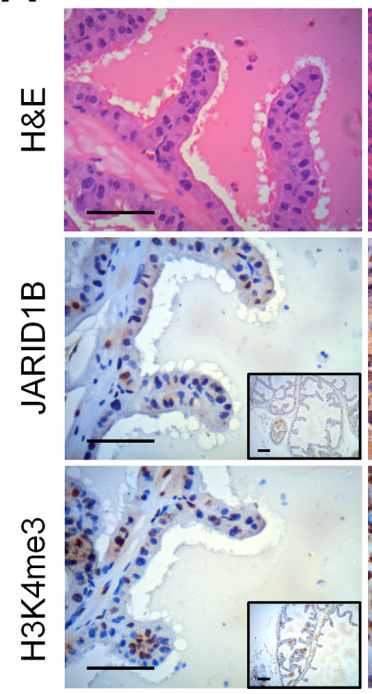

WT
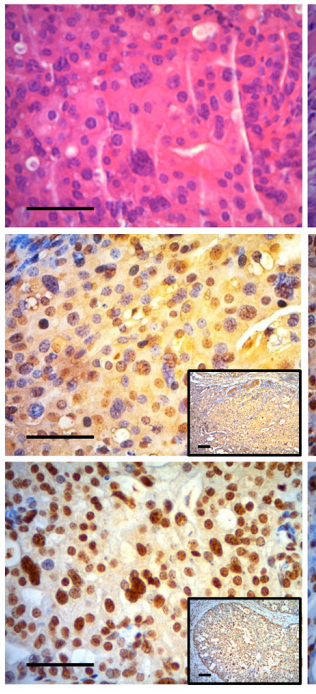

Pten ${ }^{p c-\alpha}$;

Trp53pc-/
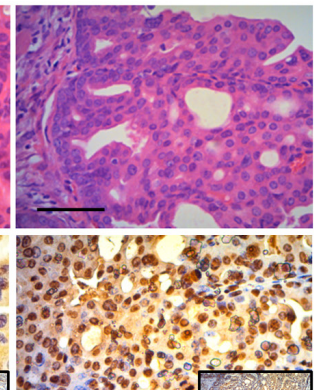

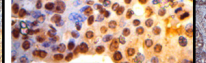
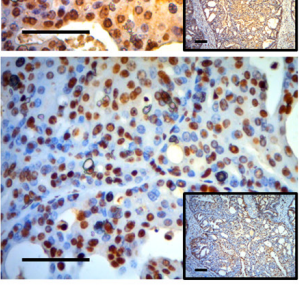

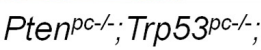

Skp2-/-
B

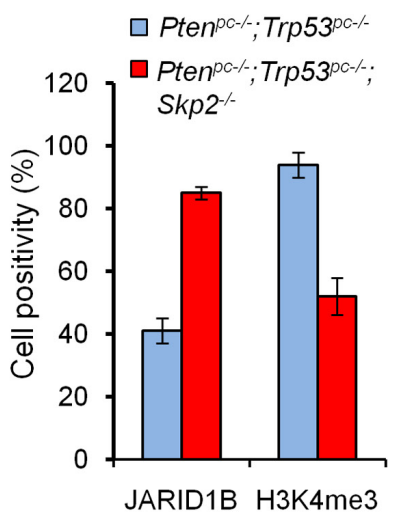

C

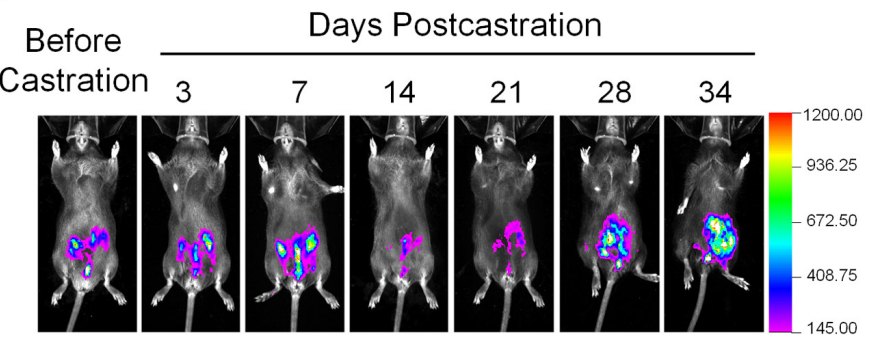

D

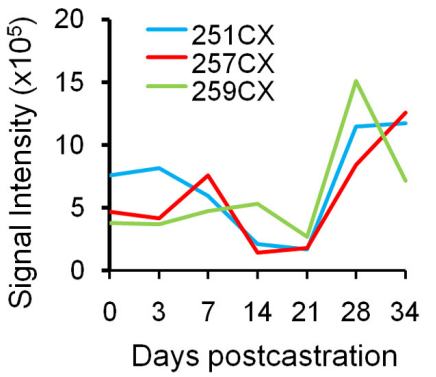

E

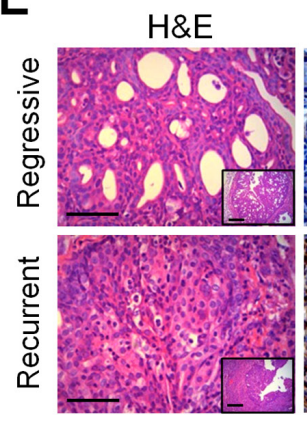

Skp2

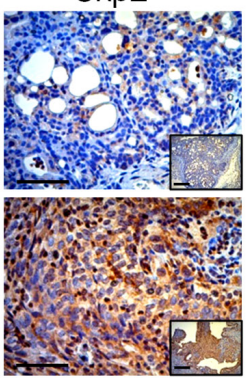

H3K4me3

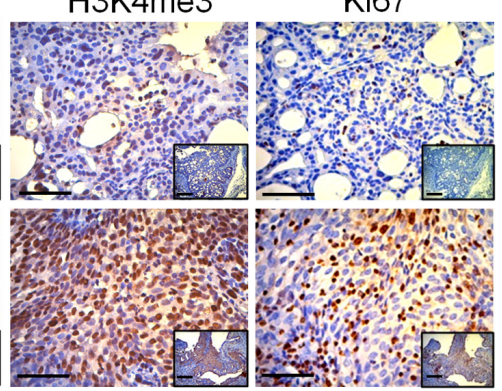

$\mathbf{F}$

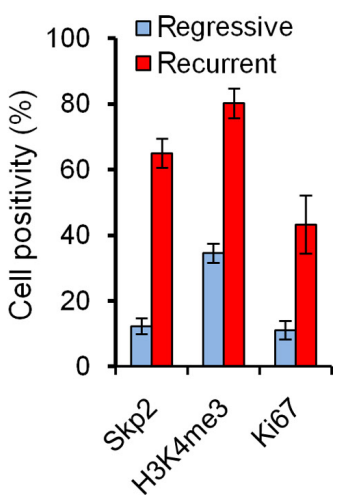

Figure 2: Skp2 inactivation decreases $\mathrm{H3K} 4 \mathrm{me} 3$ to suppress prostate cancer progression in vivo, and $\mathrm{H} 3 \mathrm{~K} 4 \mathrm{me} 3$ contributes to the recurrent growth of prostate tumors of Pten/Trp53 mice. (A) H\&E and immunohistochemical (IHC) staining for H3K4me3 and JARID1B in prostate tumors of Pten/Trp 53 and Pten/Trp53/Skp2 mutant mice. Scale bars represent $50 \mu \mathrm{m}$. (B) Quantification analysis of tumor cells positive for H3K4me3 and JARID1B. Error bars represent means \pm SD from 3 mice for each group. (C) Modeling the recurrent growth of prostate cancer in Luc/Pten/Trp53 null mice with bioluminescence imaging (BLI). The changes of BLI signals of representative Luc/Pten/Trp53 mutant mouse were monitored prior to and post castration. Mouse castration was performed at 14 weeks of age. The regressive and recurrent tumor growth were monitored weekly and defined with the change of bioluminescence intensity in modeled mice (see Supplementary Figure S2). (D) Quantification of BLI signaling changes in prostate tumors of castrated Luc/ Pten/Trp53 mutant mice $(n=3)$. The signal intensity was measured for regions of interest around the lower abdomen. (E) IHC staining of Skp2, H3K4me3 and Ki67 in regressive and recurrent lesions of prostate tumor of Luc/Pten/Trp53 mutant mice after castration. The regressive and recurrent tumor tissues of mice were collected at about 2 weeks or 3 weeks after castration, under the guidance of BLI. Scale bars represent $50 \mu \mathrm{m}$. (F) Quantification analysis of tumor cells positive for Skp2, H3K4me3 and Ki67. Error bars represent means \pm SD from 3 mice for each group. 
age when most Pten $^{p c-1}$;Trp $53^{p c-1}$ mice developed invasive cancer $[25,29,30]$. As revealed by BLI, prostate tumors of Luc/Pten/Trp 53 mutant mice displayed a striking tumor regression at 2-3 weeks post castration and then regained tumor growth (Figure 2C and 2D). All mutant mice died of (or were sacrificed due to) enlarged prostate tumors from the recurrent growth by 5-6 months of age. In order to understand the molecular profiles on the recurrent growth of $\mathrm{PCa}$, we collected the prostate tissues for histological analysis after regression or when recurrence as demonstrated by luciferase signal intensity at pertinent time points post-castration. Remarkably, IHC staining revealed that Skp2 accumulation was significantly higher in recurrent tumors as compared to regressive tumors in mice (Figure 2E and 2F), suggesting a critical role of Skp2 in CRPC. We then examined whether elevated Skp2 was associated with the dysregulations of $\mathrm{H} 3 \mathrm{~K} 4 \mathrm{me} 3$ proteins in CRPC. Importantly, H3K4me3 was indeed dramatically upregulated in recurrent tumors, when compared to that in regressive tumors (Figure $2 \mathrm{E}$ and $2 \mathrm{~F}$ ). This expression pattern of $\mathrm{H} 3 \mathrm{~K} 4 \mathrm{me} 3$ in mouse models is in agreement with the previous report on $\mathrm{H} 3 \mathrm{~K} 4 \mathrm{me} 3$ elevation in human hormone-refractory $\mathrm{PCa}[17]$. These results provide in vivo evidence to support that the dysregulation of $\mathrm{H} 3 \mathrm{~K} 4 \mathrm{me} 3$ by SKP2 contributes to CRPC growth.

\section{SKP2 determines H3K4me3 by attenuating K63-linked ubiquitination of JARID1B}

We then investigated mechanisms underlying the dysregulation of H3K4me3/JARID1B by SKP2 using human PCa cells. At first, we assessed the levels of SKP2, JARID1B and H3K4me3 proteins in several human PCa cell lines. The levels of SKP2, JARID1B and $\mathrm{H} 3 \mathrm{~K} 4 \mathrm{me} 3$ proteins are highly expressed in LNCaP, C42B, CWR22RV1 and PC3 cells (Supplementary Figure S3A). Therefore, to understand the effect of SKP2 ablation on JARID1B, we chose PC3 cells to knock down SKP2 using a lentiviral-based small hairpin RNA (shRNA) technology because PC3 cells show a high level of endogenous SKP2. As confirmed by Western blot, SKP2 knockdown completely abolished the protein expression of SKP2 in PC3-shSKP2 cells as compared to that in control cells (Figure 3A). Remarkably, H3K4me3 levels in PC3-shSKP2 cells were decreased $50 \%$ as compared to that in control cells (Figure 3A, right panel). Most importantly, SKP2 ablation resulted in a striking increase of JARID1B compared to the control. Quantification analysis revealed that JARID1B levels in PC3-shSKP2 cells were 4-fold higher than that in control cells (Figure $3 \mathrm{~A}$, right panel). These results from human PCa cells were in agreement with our data from MEFs (Figure 1F).

We reasoned that SKP2 might directly regulate the histone modification enzyme JARID1B at either the transcription or the post-transcription level. At first, we examined the changes of JARID1B mRNA upon SKP2 knockdown. Our qRT-PCR results showed that mRNA levels of JARID1B in PC3-shSKP2 cells were comparable to that in control cells (Supplementary Figure $\mathrm{S} 3 \mathrm{~B}$ ), indicating that the elevation of JARID1B protein in PCa cells upon SKP2 knockdown may be caused at the post-transcription level. We then assessed the effects of SKP2 knockdown on the stability of JARID1B protein. Surprisingly, we found that SKP2 knockdown in PC3 cells indeed resulted in a prolonged half-life of JARID1B protein. The half-life of JARID1B protein in PC3-shSKP2 cells was about $12 \mathrm{hr}$ as compared to $6 \mathrm{hr}$ in control cells (Supplementary Figure S3C).

We wished to understand whether SKP2 protein interacts with JARID1B to regulate $\mathrm{H} 3 \mathrm{~K} 4 \mathrm{me} 3$ levels in PCa cells. Immunofluorescence (IF) results revealed a co-localization of endogenous SKP2 and JARID1B proteins in nucleus of PC3 cells (Figure 3B), implicating their physical interaction. Similarly, the co-localization of JARID1B and SKP2 was found when Flag-JARID1B and Myc-SKP2 were ectopically expressed in HEK293T cells (Supplementary Figure S3D, bottom panel). Interestingly, a reverse correlation between JARID1B and SKP2 protein levels was observed endogenously in PC3 cells as well as exogenously in HEK293T cells (Supplementary Figure S3D), suggesting that SKP2 regulates JARID1B levels in cells. To confirm this interaction, HEK293T cells were cotransfected with Flag-JARID1B and Myc-SKP2 plasmids, and a co-immunoprecipitation was performed using antiFlag or anti-C-Myc. As shown, SKP2 protein was indeed detected in the immunoprecipitates with anti-Flag for JARID1B in cells cotransfected with both JARID1B and SKP2 plasmids, whereas no coimmunoprecipitation between JARID1B and SKP2 proteins was found in cells co-transfected with empty vectors or Myc-SKP2 plasmid alone (Figure 3C, top panel). In addition, JARID1B protein was detected in the immunoprecipitates when a reciprocal co-immunoprecipitation was performed using anti-Myc antibody for SKP2 (Figure 3C, bottom panel). Most importantly, the physical interaction between these two proteins was demonstrated by a co-immunoprecipitation of endogenous SKP2 and JARID1B in PC3 cells (Figure 3D). These data provided solid biochemical evidence on a physical interaction between JARID1B and SKP2 proteins in cells, supporting that the function role of SKP2 on antagonizing JARID1B protein for $\mathrm{H} 3 \mathrm{~K} 4 \mathrm{me} 3$ elevation in cancer cells.

Since SKP2 is an E3 ubiquitin ligase for the degradation of several proteins through ubiquitination $[2,3,31]$, we performed in vivo ubiquitination assay to explore whether SKP2 may also target JARID1B for the ubiquitin-mediated degradation. This would help provide a mechanistic explanation on the potential antagonizing role of SKP2 on the stability of JARID1B protein. HEK293T cells were cotransfected with SKP2, JARID1B and 
A

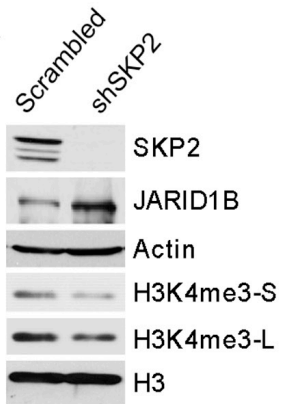

C

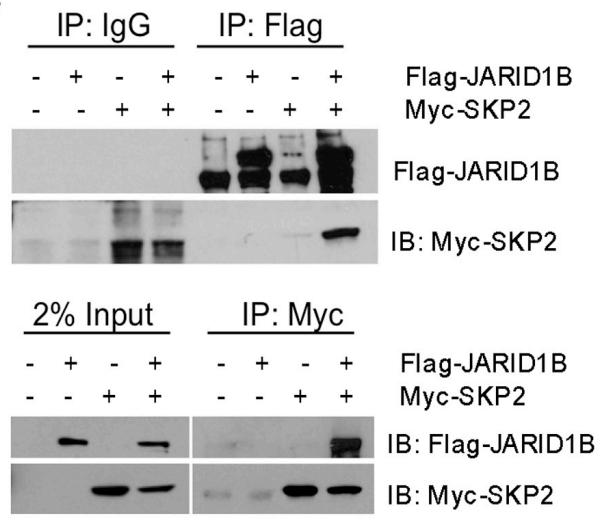

B
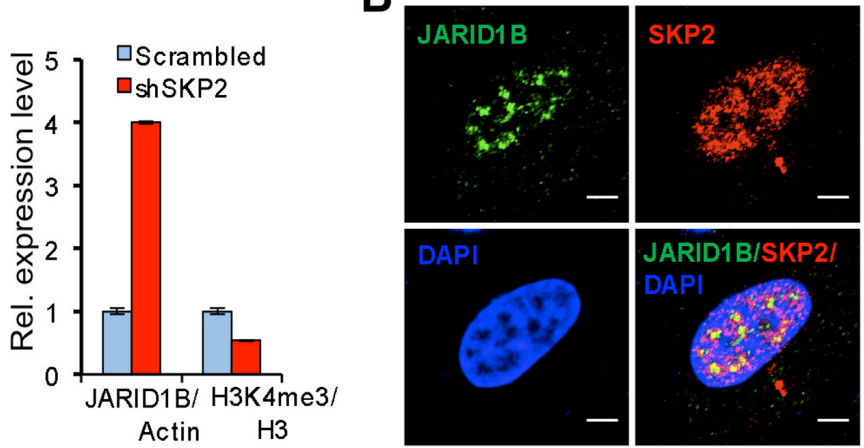

D

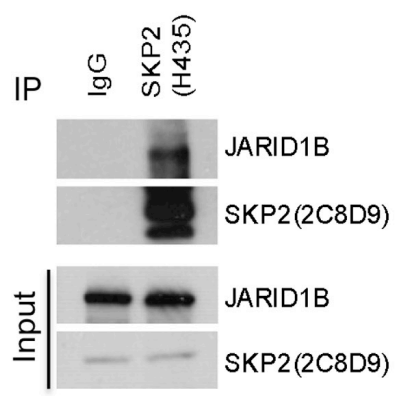

$\mathbf{F}$

E IP: Flag

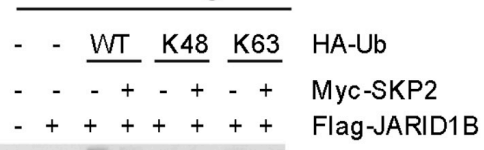

-+++++++ Flag-JARID1B

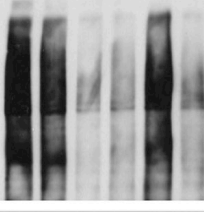

JARID1B-(Ub)n

$\leftarrow$ JARID1B

IB: HA

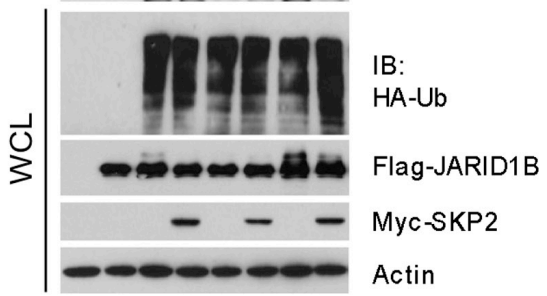

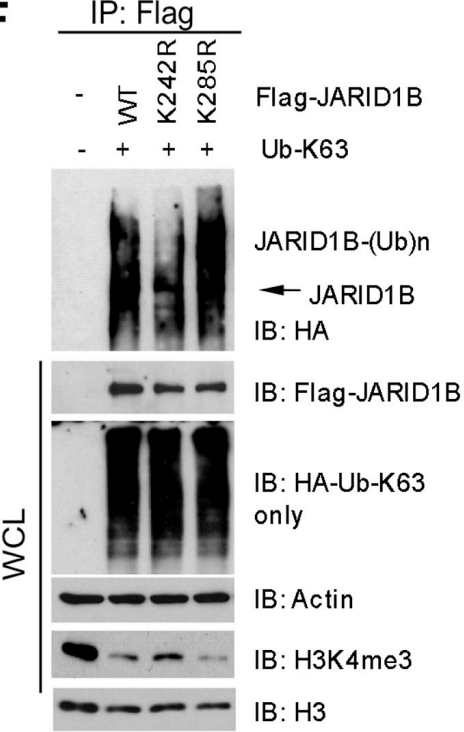

$\begin{array}{lllll}1.0 & 0.3 & 0.6 & 0.2 & \mathrm{H} 3 \mathrm{~K} 4 \mathrm{me} 3 / \mathrm{H} 3\end{array}$

Figure 3: SKP2 suppresses K63-linked ubiquitination of JARID1B to elevate H3K4me3 in human prostate cancer cells. (A) Western blot analysis shows JARID1B elevation and H3K4me3 reduction in PC3 cells upon SKP2 knockdown by shRNA. $\mathrm{S}$ - short exposure, L - long exposure. Right panel: quantification analysis on the abundance of H3K4me3 and JARID1B proteins from the left panel. Error bars represent means \pm SD. (B) Immunofluorescence images show the co-localization of endogenous SKP2 and JARID1B proteins in PC3 cells. Scale bars represent $10 \mu \mathrm{m}$. (C) Co-immunoprecipitation analysis shows a physical interaction between JARID1B and SKP2 proteins in HEK293T cells. (D) Co-immunoprecipitation analysis shows a physical interaction between endogenous JARID1B and SKP2 proteins in PC3 cells. (E) In vivo ubiquitination assay shows that a reduction of K63-linked ubiquitination of JARID1B upon addition of SKP2 in HEK293T cells. Cells were transfected with Flag-JARID1B, Myc-SKP2, along with various HA-ubiquitin (HA-Ub) constructs. K48 and K63 indicate HA-Ub-K48-only and HA-Ub-K63-only, respectively. WCL indicates whole cell lysates. (F) JARID1B mutation affects $\mathrm{H} 3 \mathrm{~K} 4 \mathrm{me} 3$ levels by altering the ubiquitination. In vivo ubiquitination assay was performed in HEK293T cells transfected with HA-Ub-K63-only, along with various JARID1B constructs (Flag-JARID1B, Flag-JARID1B-K242R and Flag-JARID1B-K285R) (see Supplementary Figure S4). Bottom panel: Western blot analysis shows the effects of JARID1B WT and JARID1B mutants on the changes of endogenous protein levels of H3K4me3 through the K63-linked ubiquitination in cells. WCL indicates the whole cell lysates. 
HA-tagged ubiquitin or ubiquitin mutants including K48only and K63-only ubiquitin plasmids. Surprisingly, our results revealed that the polyubiquitination of JARID1B protein was conjugated through ubiquitin at lysine 63 instead of lysine 48, the common form of polyubiquitination for proteasome-dependent protein degradation (Figure 3E). Surprisingly, we observed that addition of SKP2 failed to promote, but reduced, JARID1B ubiquitination contributed by WT ubiquitin and ubiquitin K63-only mutant. Lysine 48(K48)-linked ubiquitination is generally contributing to protein degradation through ubiquitin-mediated proteasome pathway, while lysine 63(K63)-linked ubiquitination is responsible for various protein functions including kinase activity and protein trafficking [32]. Our results suggest that SKP2 may have a novel regulatory role on K63-linked polyubiquitination of JARID1B, in addition to its E3 ubiquitin ligase activity.

To define the biological relevance of K63-linked JARID1B ubiquitination in PCa cells, we decided to generate JARID1B mutants to see the impact on $\mathrm{H} 3 \mathrm{~K} 4 \mathrm{me} 3$ levels, the enzymatic product of JARID1B. UbPred program predicted that lysine residues 242 and 285 are potential ubiquitination sites of JARID1B (Supplementary Figure S4) [33]. We generated JARID1B mutants by sitedirected mutagenesis and then performed in vivo K63linked ubiquitination assay. Our results demonstrated that JARID1B mutant at lysine 242 (K242R) but not at lysine 285 (K285R) resulted in a dramatic decrease of K63-linked ubiquitination of JARID1B, when compared to that of wild type JARID1B (WT) (Figure 3F), suggesting lysine 242 in JARID1B may be one of the major conjugation sites for polyubiquitination through lysine 63 of ubiquitin. Furthermore, we examined the effects of K63-linked polyubiquitination of JARID1B on the levels of $\mathrm{H} 3 \mathrm{~K} 4 \mathrm{me} 3$. Our results showed that overexpression of JARID1B WT or K285R mutant, resulted in a dramatic reduction of $\mathrm{H} 3 \mathrm{~K} 4 \mathrm{me} 3$ level to $30 \%$ and $20 \%$, respectively, as compared to the control, the endogenous level of H3K4me3 in HEK293T cells. In contrast, K242R mutant of JARID1B only reduced H3K4me3 level to $60 \%$ compared to the control, resulting in 2 or 3 -fold elevation of $\mathrm{H} 3 \mathrm{~K} 4 \mathrm{me} 3$ levels than that of JARID1BWT and JARID1B-K285R (Figure 3F). This result is consistent with the inhibition of K63-linked ubiquitination of JARID1B by K242R mutation in the presence of Ub-K63-only proteins. These lines of biochemical evidence indicate that K63-linked ubiquitination of JARID1B is required for its enzymatic activity to demethylate H3K4me3.

\section{SKP2 downregulates TRAF6-mediated ubiquitination of JARID1B}

Since JARID1B ubiquitination is not directly catalyzed by SKP2, we deduced that SKP2 might regulate other E3 ubiquitin ligases responsible for the K63- linked ubiquitination of JARID1B to indirectly affect $\mathrm{H} 3 \mathrm{~K} 4 \mathrm{me} 3$ levels. We reasoned that TRAF6 is a candidate E3 ubiquitin ligase for K63-linked ubiquitination of JARID1B, as it is mainly involved in the synthesis of polyubiquitin chains linked through ubiquitin lysine 63 . Literature reports that TRAF6 is required to promote TGF- $\beta$ induced apoptosis of PCa cells through activation of TGF- $\beta$-Associate Kinase 1 (TAK1) in TGF- $\beta$ signaling pathway $[34,35]$. In silico amino acid sequence analysis revealed that JARID1B contains three potential consensus TRAF6 binding motifs (PxExxAr/Ac), which are adjacent to the ubiquitination site of JARID1B at lysine 242 (Supplementary Figure S5A). IF assay showed a colocalization of TRAF6 and JARID1B proteins, suggesting the existence of their physical interaction (Figure 4A). We next performed reciprocal co-immunoprecipitation assays with anti-TRAF6 or anti-JARID1B antibody using lystates of PC3 cells that contain both endogenous JARID1B and TRAF6. Our data showed that these two proteins physically interact, suggesting that TRAF6 may be an E3 ubiquitin ligase for the K63- linked ubiquitination of JARID1B (Figure 4B and 4C). To confirm this possibility, we performed in vivo ubiquitination assay with HEK293T cells that were cotransfected with TRAF6, JARID1B or JARID1B mutants and ubiquitin-K63-only mutant plasmids, in the absence or the presence of SKP2. As expected, TRAF6 dramatically increased the K63-linked polyubqiuitination of JARID1B, and addition of SKP2 resulted in a striking reduction of the polyubiquitination (Figure 4D). Furthermore, JARID1B K242R mutation abrogated TRAF6-mediated ubiquitination of JARID1B (Figure 4E).

We investigated the relationship between SKP2 and TRAF6 to elucidate the role of SKP2 on TRAF6mediated JARID1B ubiquitination in PCa cells. Western blot analysis revealed that TRAF6 protein levels were markedly elevated in PC3 cells upon SKP2 knockdown as compared to the control (Supplementary Figure S5B). In addition, IHC results also showed that TRAF6 levels were strikingly increased in prostate tumors of $\mathrm{Pten}^{\mathrm{pc-1} \text {; }}$ $\operatorname{Trp} 53^{p c-1}$; Skp2 $2^{-/}$mice, as compared to that of Pten ${ }^{p c-/}$; Trp $53^{p c-1}$ mice (Supplementary Figure S5C). These data support that SKP2 maintains a low level of TRAF6 protein both in vitro and in vivo. In summary, our results disclose a novel mechanism by which SKP2 indirectly inhibits the K63-linked ubiquitination of JARID1B via E3 ubiquitin ligase TRAF6.

\section{SKP2 deficiency promotes ubiquitinated JARID1B in nucleolus of PCa cells for senescence in vitro and in vivo}

Our finding on the K63-linked ubiquitination of JARID1B mediated by SKP2 promoted us to hypothesize that SKP2 may also contribute to histone modifications by regulating protein trafficking of JARID1B in PCa cells. 
A

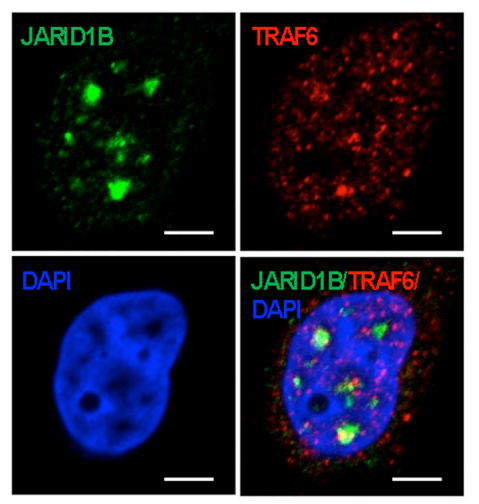

B
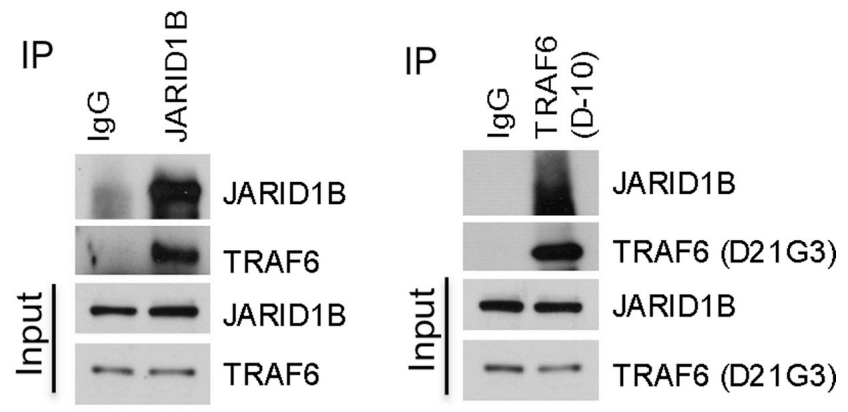
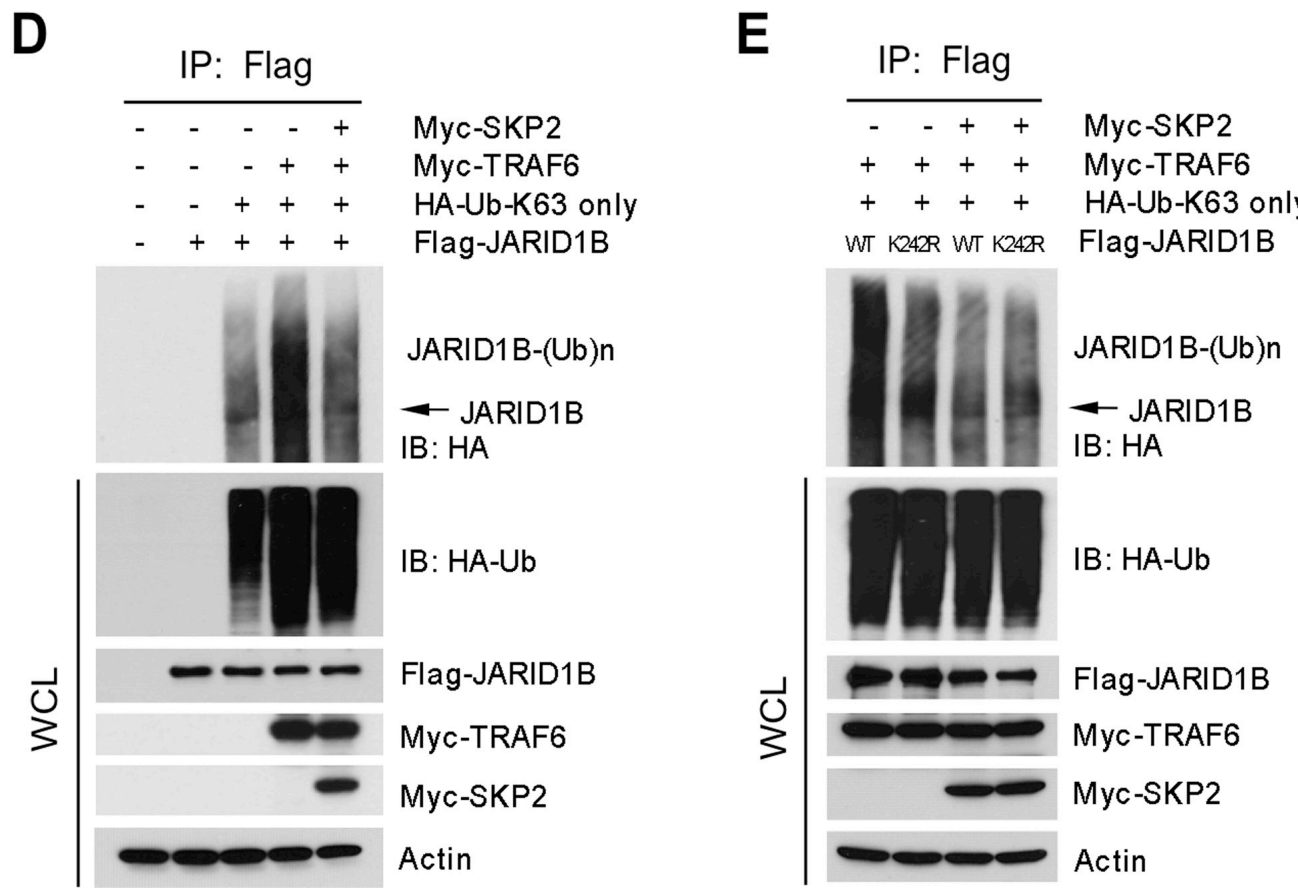

Figure 4: SKP2 regulates the ubiquitination of JARID1B through TRAF6. (A) Immunofluorescence images show a colocalization of endogenous JARID1B and TRAF6 in PC3 cells. Scale bars represent $10 \mu \mathrm{m}$. (B) and (C) Co-immunoprecipitation analysis shows that endogenous TRAF6 physically interacts with JARID1B in PC3 cells, as shown by reciprocal co-immunoprecipitation between the two proteins (Also see Supplementary Figure S5). (D) In vivo ubiquitination assay shows that TRAF6 increases K63-linked ubiquitination of JARID1B and SKP2 inhibits TRAF6-mediated JARID1B ubiquitination. Cells were transfected with Flag-JARID1B, HA-Ub-K63-only, Myc-TRAF6 and Myc-SKP2 constructs as indicated. WCL indicates the whole cell lysates. (E) TRAF6 mediates JARID1B ubiquitination through lysine residue 242. HEK293T cells were transfected with Flag-JARID1B WT or Flag-JARID1B-K242R, HA-Ub-K63-only, MycTRAF6 and Myc-SKP2 plasmids as indicated. In vivo ubiquitination assay was performed in a standard procedure. WCL indicates the whole cell lysates.

To test this hypothesis, we performed IF analyses to inquire the subcellular localization of JARID1B in PC3-shSKP2 cells compared to that in PC3-scrambled cells. Unexpectedly, we observed an altered distribution of JARID1B protein in nucleus of PC3 cells upon SKP2 knockdown, indicating that the trafficking of JARID1B protein was restrained inside nucleus, very little to cytoplasm (Figure 5A and Supplementary Figure S6A and S8A). Fibrillarin (FBL), a major component of ribonuceloproteins in nucleolus, is recently reported to associate with histone modifications [36]. Therefore, we decided to examine whether JARID1B protein shuttled to nucleoli of PC3 cells upon SKP2 knockdown. As shown, we found that JARID1B is predominantly localized in the nucleoli of PC3-shSKP2 cells upon SKP2 knockdown as evidenced by its co-localization with FBL, whereas JARID1B is largely localized in the nuceloplasm of PC3-scrambled cells 
(Figure 5A and Supplementary Figure S6A, arrows in white color). Moreover, we sought for the nucleolar localization signal (NoLS) in JARID1B protein, and found four potential NoLS sequences predicted by NOD program (Supplementary Figure S7). For a validation of NOD program, human p14ARF-a known nucleolar protein was tested as a control, and genuine NoLS signal sequences were identified (Supplementary Figure S7) [37]. To confirm that JARID1B protein in nucleolus still remains in the K63-linked ubiquitination, we performed IF staining with K63-Ub antibody. Our results showed that K63-Ub was co-localized with JARID1B in the nucleolus of PC3-shSKP2 cells, but not in that of PC3-scrambled cells (Figure 5B and Supplementary Figure S8B). These results indicate that the K63-linked ubiquitination of JARID1B may be required for JARID1B protein to shuttle to nucleoli for biological functions upon SKP2 knockdown.

SKP2 deficiency induces cellular senescence in contexts of oncogenic insults [8], and recently JARID1B is associated with cellular senescence in colorectal cancer and retinoblastoma cells [38, 39]. We reasoned that K63-linked ubiquitination of JARID1B accumulated in nucleolus may contribute to SKP2-deficient induced cellular senescence. To test this hypothesis, we assessed the protein level of $\beta$-galactosidase, a senescence marker, in PC3-shSKP2 cells. As predicted, a striking increase of $\beta$-galactosidase was found in PC3-shSKP2 cells, as compared to that in PC3scrambled cells (Figure 5C), in agreement with literature that SKP2 deficiency induces cellular senescence. Importantly, IF results revealed that a remarkable accumulation of JARID1B protein was found in nucleolus of senescent PC3-shSKP2 cells but not in non-senescent PC3-srambled cells (Figure 5D). These results indicate that nucleolar JARID1B may contribute to the induction of cellular senescence in human PCa cells. Our findings in vitro on the SKP2 deficiency-promoted shuttling of JARID1B protein encouraged us to validate the phenomenon in prostate tumors of mice. Our results showed that JARID1B and FBL proteins are co-localized in a majority of epithelial cells in prostate tumors of Pten ${ }^{p c-}$; Trp $53^{p c-1}$; Skp $2^{-\leftarrow}$ mice, but not in that of Pten ${ }^{p c-1}$; Trp $53^{p c-1}$ mice (Figure 5E and Supplementary Figure S6B). Similarly, the co-localization of JARID1B and $\mathrm{K} 63-\mathrm{Ub}$ proteins was also found in prostate epithelial cells of Pten $^{p c-/}$; Trp $53^{p c-/}$; Skp $2^{-\leftarrow}$ mice (Supplementary Figure S8C). The cellular senescence was detected in prostate tumors of $\operatorname{Pten}^{p c-/}$; Trp $53^{p c-/} ;$ Skp $2^{--}$mice, but not in that of Pten ${ }^{p c-1}$; Trp $53^{p c-1}$ mice (Figure 5F).

Taken together, we demonstrated that SKP2 restricts JARID1B trafficking to nucleoli of PCa cells through attenuating its K63-linked ubiquitination. The K63linked ubiquitination procedure is likely required for the nucleolar shuttling to maintain the function of JARID1B for demethylation of $\mathrm{H} 3 \mathrm{~K} 4 \mathrm{me} 3$ and for the regulation of cellular senescence in PCa cells. SKP2 deficiency suppresses PCa by altering JARID1B ubiquitination, shuttling, and thus contributing to epigenetic modifications of $\mathrm{H} 3 \mathrm{~K} 4 \mathrm{me} 3$ and cellular homeostasis.

\section{H3K4me3 levels are positively correlated with SKP2 expression in human prostate cancer}

To understand the correlation between SKP2 and $\mathrm{H} 3 \mathrm{~K} 4 \mathrm{me} 3$ as well as the relevance in human $\mathrm{PCa}$, we performed IHC staining of SKP2 and H3K4me3 in human prostate tissue microarrays (TMA) consisting of cancer and normal cases. The elevation of SKP2 protein was found in both cytoplasm and nucleus, while H3K4me3 was primarily accumulated in the nucleus of cancer cells (Figure 6A). In addition, the correlation between SKP2 and H3K4me3 levels was assessed by Pearson correlation test, with grades taken as continuous variables, which was further validated by Chi-square tests. Results from both statistical analyses indicated that SKP2 expression was positively correlated with $\mathrm{H} 3 \mathrm{~K} 4 \mathrm{me} 3$ levels (Pearson correlation coefficient $=0.4693, P=0.0044 ; \chi 2$ test, $P=0.0006$ ) in PCa (Figure 6B and Supplementary Table S2), when graded in intensity staining scores, respectively. These data suggest the deregulation of SKP2 and $\mathrm{H} 3 \mathrm{~K} 4 \mathrm{me} 3$ plays essential roles in human PCa.

\section{DISCUSSION}

Aberrant elevation of histone post-translational modifications is recognized to make important contributions to the initiation and progression of human cancers by altering the expression profile of multiple genes $[18,27]$. The dysregulation of H3K4me3, one of the most important histone methylations, has been found to associate with the development of cancers including $\mathrm{PCa}$ [18]. Given that H3K4me3 levels are mainly determined by JARID1B, it is crucial to understand molecular mechanisms on the regulation of JARID1B and the relevance in PCa. Yet, due to the limits of current technology and lack of authentic mouse models, dysregulation of H3K4me3 and JARID1B in vivo upon oncogenic insults remains poorly understood [19]. Our studies with in vitro and in vivo evidence added valuable knowledge to decode this phenomenon in 3 ways: 1) Skp2 ablation results in a reduction of $\mathrm{H} 3 \mathrm{~K} 4$ trimethylation and a suppression of cancer progression; 2) JARID1B activity requires its K63-linked ubiquitination, which is suppressed by elevated SKP2 through regulating E3 ubiquitin ligase TRAF6 in PCa cells; and 3) K63-linked ubiquitination of JARID1B may be required for protein trafficking into nucleolus to control cellular homeostasis through senescence mechanism. Our results provide new and valuable mechanistic insights into understanding the regulation of $\mathrm{H} 3 \mathrm{~K} 4 \mathrm{me} 3$ and JARID1B and further effects of oncogenic protein SKP2 on PCa. 
A

PC3 cells
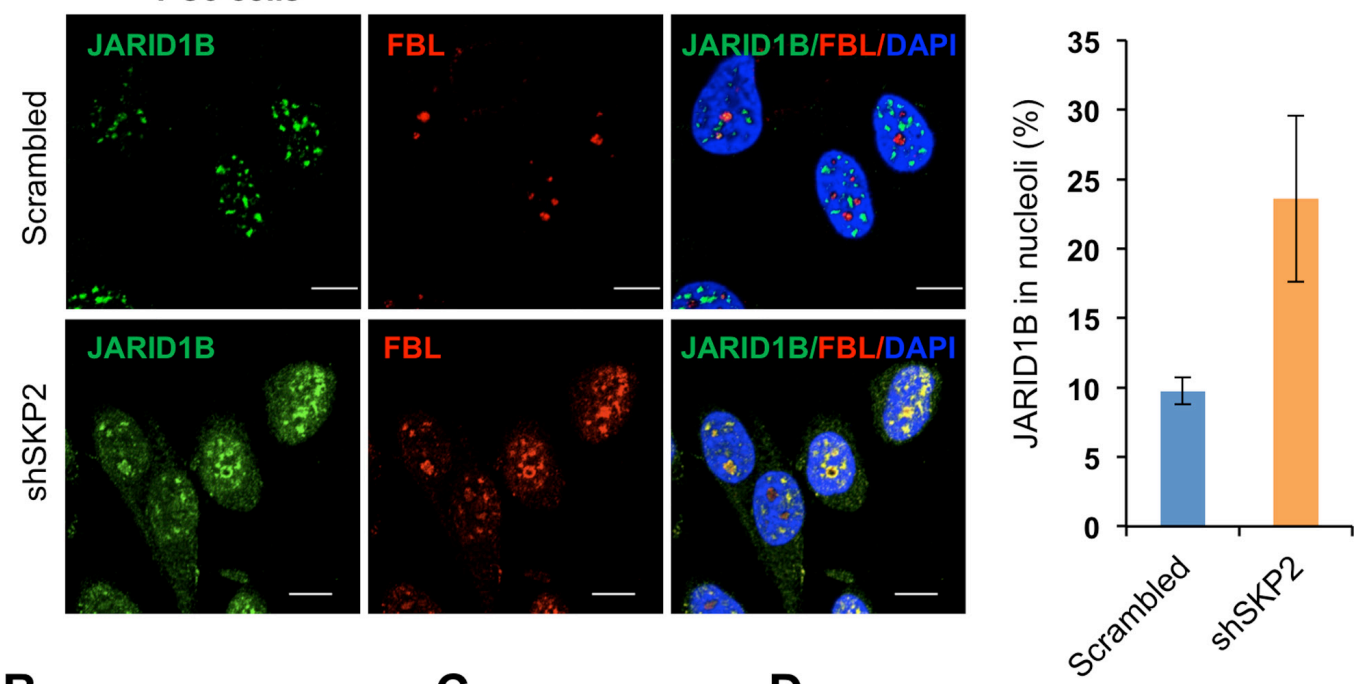

B

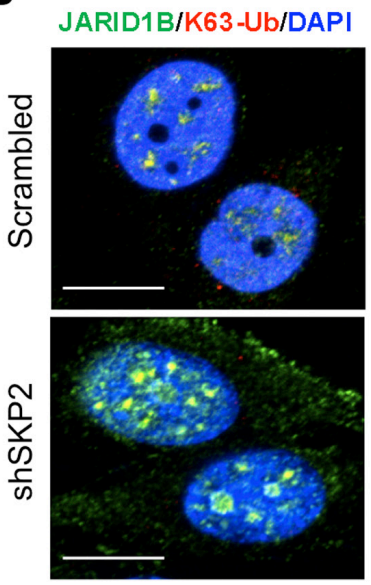

C

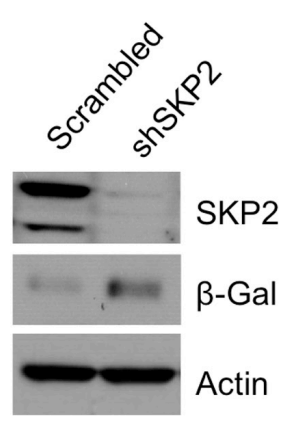

D

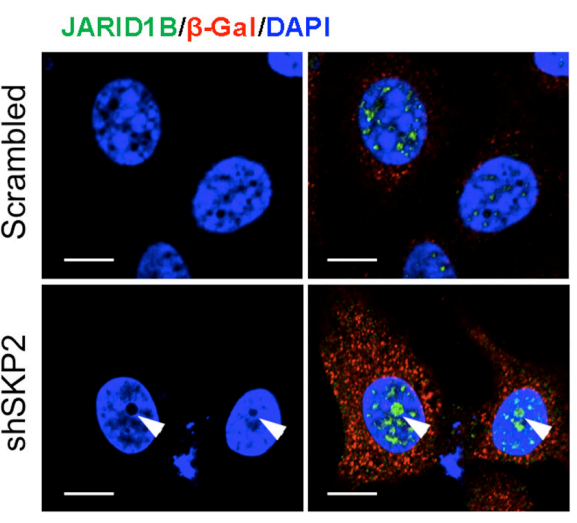

E

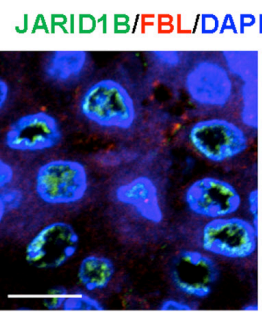

Pten $^{\text {pat; }}$

Trp53 $3^{p-1-}$

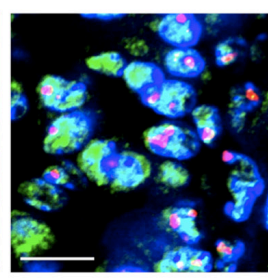

Pten $^{p c-1-}$ Trp 53 $3^{p c-1-}$ Skp2 ${ }^{-1-}$

F

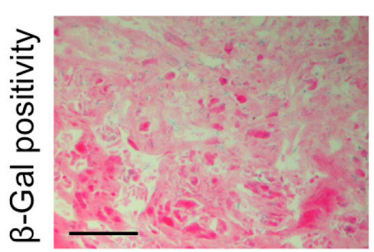

$P^{\text {Pten }}{ }^{p-\alpha}$

Trp53 $50-$ -

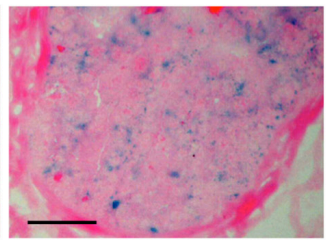

Pten ${ }^{p c--;}$ Trp53 $50-$;

Skp2--

Figure 5: SKP2 inactivation induces an accumulation of ubiquitinated JARID1B in nucleolus of cells in vitro and in vivo for cellular senescence. (A) Immunofluorescence (IF) images show a co-localization of endogenous JARID1B and Fibrillarin (FBL) in nucleoli of PC3 cells upon SKP2 knockdown (Also see Supplementary Figure S6A). FBL indicates a nucleolar marker. Right panel: quantification of PC3 cells showing an increase of JARID1B localization in nucleolus. Error bars represent means \pm SD. (B) IF images show a co-localization of endogenous JARID1B and K63-Ub in nucleoli of PC3 cells upon SKP2 knockdown. (C) Western blotting assay shows an increase of $\beta$-galactosidase ( $\beta$-Gal) in PC3 cells upon SKP2 knockdown. (D) IF images show JARID1B in nucleolus as indicated by arrows and $\beta$-Gal in cytoplasm in senescent cells upon SKP2 knockdown. (E) The co-localization of endogenous JARID1B and Fibrillarin (FBL) in nucleoli of prostate tissues in $\operatorname{Pten}^{p c-/} ; \operatorname{Tr} p 53^{p c-/} ;$ Skp $2^{--}$mutant mice (Also see Supplementary Figure S6B). Scale

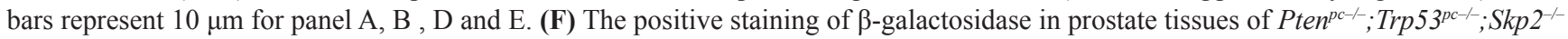
mice but not in that of $\operatorname{Pten}^{p c-/} ; \operatorname{Trp} 53^{p c-/-}$ mice. Scale bars represent $50 \mu \mathrm{m}$. 
A

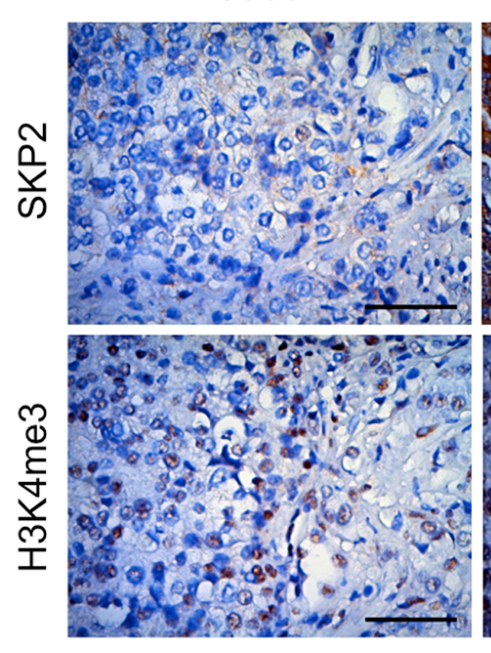

Case 2

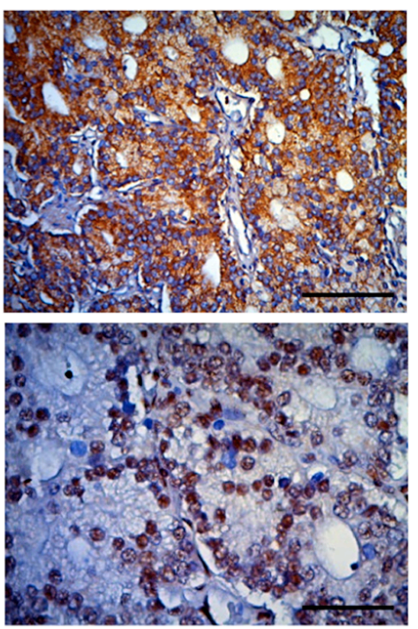

B

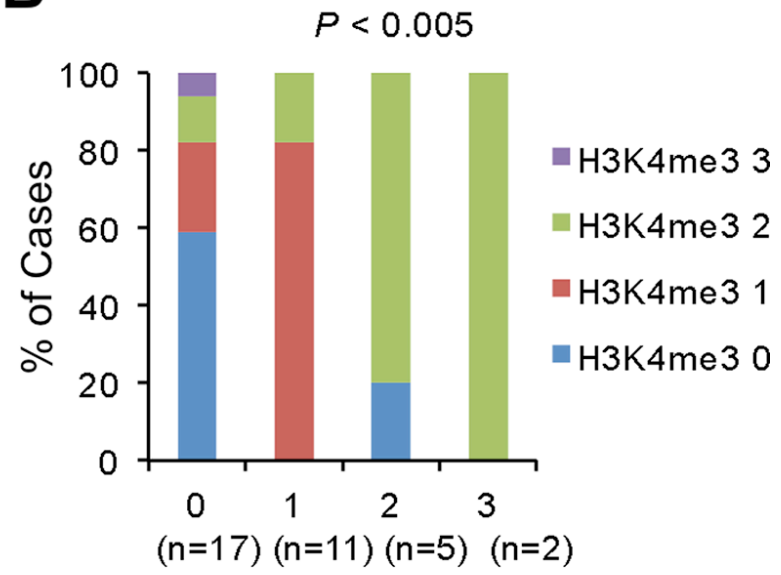

SKP2 detection level

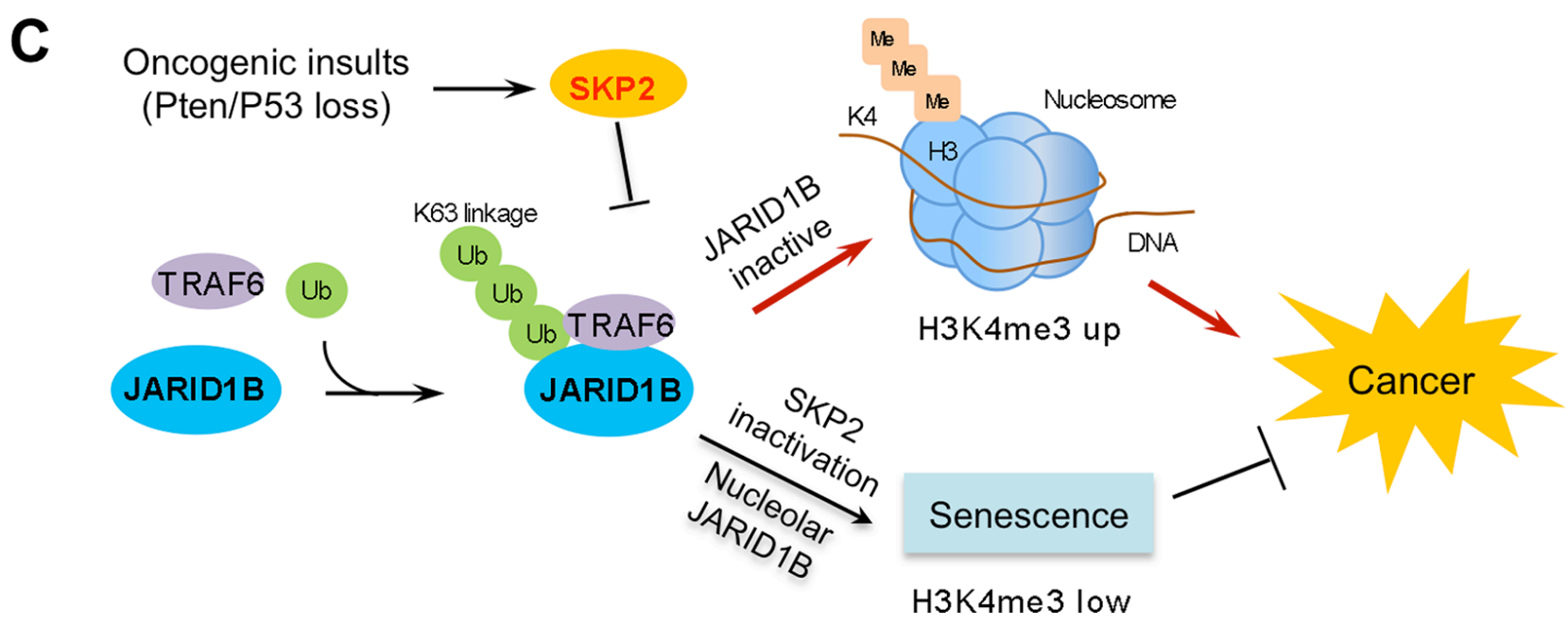

Figure 6: SKP2 positively correlates with H3K4me3 in human prostate cancer specimens. (A) Immunohistochemical staining on SKP2 and H3K4me3 in human prostate array tissues. Scale bars represent $50 \mu \mathrm{m}$. (B) Statistical analysis of the prostate tissue microarray stained with SKP2 and H3K4me3 antibodies. The percentages of different H3K4me3 levels were calculated for each level of SKP2 protein in 35 cases of human PCa specimens. SKP2 and H3K4me3 levels were graded as 0, 1, 2 and 3 by intensity scores. The $\mathrm{H} 3 \mathrm{~K} 4 \mathrm{me} 3$ grades are color-coded. Numbers in parenthesis represent sample sizes. The statistical significance was determined by ChiSquare test (Table S2). (C) A working model for the role of SKP2 on epigenetic regulation of JARID1B and H3K4me3 in PCa. SKP2 suppresses the activity of JARID1B by reducing its K63-linked ubiquitination through TRAF6 under oncogenic stimulation, leading to an elevated H3K4me3 thus contributes to PCa. SKP2 deficiency increases JARID1B transportation to nucleolus of cells through an increase of its ubiquitination, resulting in an induction of cellular senescence to suppress PCa tumorigenesis.

Both SKP2 elevation and aberrant histone modifications are frequently detected in CRPC and recognized as important markers for cancers [27, 40, 41]. SKP2 is required to maintain the normal cell cycle and its inactivation results in cell cycle arrest [2]. Importantly, upon oncogenic insults, SKP2 inactivation drives cell cycle arrest to irreversible senescence (also termed as geroconversion) [8, 42]. However, the connection between SKP2 elevation and histone modifications remains unclear [18]. In this study, we applied genetic approaches to dissect the molecular contributions of SKP2 to epigenetic alterations in prostate tumorigenesis. This notion is supported with in vitro evidence that Skp2 deficiency reduces cell proliferation, transformation and migration of Pten/Trp53 double null MEFs (Figure 1), and with in vivo evidence that SKP2 deficiency constrains the initiation and growth of prostate tumors of $\mathrm{Pten}^{p c-1}$; Trp $53^{p c-1}$ mice (Figure 1 and Supplementary Figure S1). Importantly, we discovered that Skp2 elevation was highly correlated with the increased levels of H3K4me3 in primary (Data 
not shown) and recurrent $\mathrm{PCa}$ tissues in mice upon oncogenic stimulation (Pten/Trp53 loss) (Figure 2E), whereas Skp2 ablation dramatically reduced $\mathrm{H} 3 \mathrm{~K} 4 \mathrm{me} 3$ (Figure $1 \mathrm{~F}$ and 2A). These results indicate the abnormal elevation of H3K4me3 depends on Skp2, at least in Pten/ Trp53 null background, which implicating that SKP2 plays an essential role in dysregulation of $\mathrm{H} 3 \mathrm{~K} 4 \mathrm{me} 3$ for the development and progression of cancers. Our findings are in harmony with previous reports that SKP2 has a critical pro-oncogenic function for the development and progression of cancers including $\mathrm{PCa}$, through affiliating oncogenic signaling pathways such as RhoA, c-Myc and $\operatorname{Ras}[6,8,10]$. It will be worthy to evaluate if the dysregulation of $\mathrm{H} 3 \mathrm{~K} 4$ trimethylation depends on Skp2 in other mouse models of cancers, and furthermore the relevance in mTOR signaling pathway [43].

Methylation, acetylation and phosphorylation of histones contribute to cancers and diseases in humans by synchronizing the epigenetic modification cascades to mediate the functions of oncogenes and tumor suppressor genes in cells [44-47]. On the other hand, aberrant histone modifications are substantially associated with aberrant expression and mutations of epigenetic modification regulators in human cancers [17, 18]. We revealed a novel molecular mechanism in which SKP2 determines the levels of $\mathrm{H} 3 \mathrm{~K} 4 \mathrm{me} 3$ by mediating $\mathrm{K} 63$ linked ubiquitination of JARID1B, a key demethylase of $\mathrm{H} 3 \mathrm{~K} 4 \mathrm{me} 3$, through regulating E3 ubiquitin ligase TRAF6. The K63-linked ubiquitinated form of JARID1B interacts with SKP2 in the nucleoplasm of cells when SKP2 is present or elevated, but sharply increases and shuttles to the nucleolus of PCa cells upon SKP2 ablation (Figure 5 and Supplementary Figure S6 and S8). Most recently, Tessarz et al. showed that nucleolus is involved in histone modifications [36], so it is likely that JARID1B/ $\mathrm{H} 3 \mathrm{~K} 4 \mathrm{me} 3$ coupling requires Fibrillarin in nucleolus under this setting. Paradoxically, the protein level of JARID1B increases upon SKP2 loss in both MEFs and human PCa cells, suggesting the JARID1B protein stability may be mediated by SKP2 through ubiquitination mechanisms independent of lysine 48. For example, literature reports that sumoylated JARID1B/KDM5B on lysine 242 can be marked by E3 SUMO ligase RNF4 for proteasome mediated degradation [48]. In this study, we observed that the lysine 242 of JARID1B is ubiquitinated through ubiquitin K63-linkage, suggesting that JARID1B protein may be differentially regulated by synchronization of the ubiquitination and sumoylation machineries in cancers. It is likely that an increase of JARID1B ubiquitination in the absence of SKP2 will competitively decrease the free lysine 242 for the sumoylation-mediated degradation by RNF4.

JARID1B/KDM5B/PLU1, the H3K4me3/2 demethylase, is frequently elevated in advanced PCa [19, 20,49] and associated with multidrug resistance [50], underscoring its potential oncogenic roles in cancers. The mechanisms leading to JARID1B elevation and possible driving pathways to cancers, in addition to its demethylase function, are still paucity of understanding. In our study, we unveiled in vitro and in vivo that JARID1B was regulated by SKP2 at a post-translational fashion using several biological systems including human PCa cells, MEFs, and mouse models. Our results showed that SKP2 had significant effects on JARID1B activity and H3K4me3 levels. SKP2 knockdown or deficiency results in an increase of JARID1B protein level, which partially in line with the literature that SKP2 is an important component of SCF complex functioning for protein degradation [7, 51]. Importantly, we found that SKP2 elevation is positively correlated with $\mathrm{H} 3 \mathrm{~K} 4 \mathrm{me} 3$ in $\mathrm{PCa}$ specimens (Figure $6 \mathrm{~A}$ and $6 \mathrm{~B}$ ). Taken together, our study revealed a novel function of SKP2 in mediating JARID1B for histone methylations (Figure 6C), and further investigation is needed to decipher the molecular interactions among SKP2, TRAF6 and JARID1B proteins.

In short, our studies revealed a novel mechanism in which JARID1B is regulated by SKP2 through K63-linked ubiquitination, which leading to aberrant alterations of $\mathrm{H} 3 \mathrm{~K} 4 \mathrm{me} 3$ in turmorigenesis and CRPC. Furthermore, our results, together with previous reports, support that Skp2 deficiency restrains cell proliferation and tumor progression by triggering cellular senescence [8], chromatin remodeling and histone modifications [39]. Our findings on SKP2 and JARID1B suggest that a combinatory targeting on SKP2 and JARID1B may be more potent chemotherapy for CRPC treatment.

\section{MATERIALS AND METHODS}

\section{Mutant mice, genotyping and tumor analysis}

Pten $^{\text {loxP/loxP }} ;$ Trp53 5 loxP/loxP. Skp2 $2^{-/}$and ProbasinCre 4 mutant mice were generated and maintained as previously described $[8,25]$. Briefly, in order to obtain desired genotypes of compound mutant mice, female mice carrying alleles of LoxPten, LoxTrp53, and Skp2 $2^{+-}$ were crossed with male mice carrying alleles of LoxPten, LoxTrp53, Skp2 $2^{+-}$and Probasin-Cre4 to produce conditional double knockout mutants $P$ ten ${ }^{\text {loxPloxP }}$; Trp $53^{\text {loxP/ }}$ loxP ; Probasin-Cre4 (referred to Pten ${ }^{p c-1}$; Trp53 $3^{p c--}$ ) and conditional triple knockout mutants $P$ ten ${ }^{\text {loxPloxP }} ; \operatorname{Trp} 53^{\text {loxP/ }}$ loxP ; Skp2 ${ }^{--\alpha}$; Probasin-Cre (referred to Pten ${ }^{p c-1}$; Trp53 ${ }^{p c-1}$; $S k p 2^{-/}$or Pten/Trp53/Skp2). To generate the prostatespecific luciferase Pten/Trp53 double null mutant mice, luciferase transgenic female mice ( $\left.L u c^{R O S A 26}{ }^{L S L}\right)$ were crossed with Pten ${ }^{\text {loxP } /+} ;$ Trp $53^{\text {loxP/loxP }} ;$ Probasin-Cre 4 male mice. Their F2 offspring was further used to generate Luc $^{\text {LSL }}$; Pten ${ }^{\text {loxP/loxP }}$; Trp53 $3^{\text {loxP/loxP }} ;$ Probasin-Cre4 (referred to $\mathrm{Luc}^{p c+}$; Pten ${ }^{p c-/}$; Trp53 $3^{p c-/}$ or Luc/Pten/Trp53) compound mutants. All experimental animals were kept in a mixed genetic background of C57BL/6J X 129sv $\mathrm{X}$ DBA2, and animal experiments were conducted in 
accordance with an IACUC-approved protocol at Meharry Medical College.

All genotypes were verified by performing polymerase chain reaction (PCR) with DNA extracted from mouse tails according to protocols previously described $[8,25]$. Briefly, mouse tail tissues at $0.5 \mathrm{~cm}$ in length were digested in a volume of $300 \mu$ lysis buffer containing Proteinase K $(350 \mu \mathrm{g} / \mathrm{ml})$ over night at $55^{\circ} \mathrm{C}$, and DNA was precipitated with 2 volumes of ethanol and then suspended in $500 \mu \mathrm{l}$ of $\mathrm{ddH}_{2} \mathrm{O}$ for use. Primers used for genotyping PCR were previously described and listed in Supplementary Table S1. PCR programs were run as $95^{\circ} \mathrm{C}$ for $4 \mathrm{~min}$, then $95^{\circ} \mathrm{C} 30 \mathrm{~s}, 57^{\circ} \mathrm{C} 1 \mathrm{~min}, 72^{\circ} \mathrm{C} 1 \mathrm{~min}$ for 34 cycles, with a final elongation at $72^{\circ} \mathrm{C}$ for $7 \mathrm{~min}$ in a BioRad thermal cycler.

Mice in indicated genotypes were sacrificed at 3 or 6 months of age (mice with enlarged tumors were sacrificed according to the IACUC protocol), and their anterior prostate (AP) tissues or tumors were dissected and weighed. Tissues were fixed in 10\% neutral-buffered formalin (Sigma) over night and then preserved in $70 \%$ ethanol at $4^{\circ} \mathrm{C}$. Fixed tissues were further processed by ethanol dehydration and embedded in paraffin according to standard protocols (Histoserv Inc., Gaithersburg, MD).

\section{Cell proliferation, transformation and invasion assays}

Mouse embryonic fibroblasts (MEFs) were prepared from individual embryos of various genotypes at E13.5day according to the procedure previously described $[25,26]$. MEFs were cultured in Dulbecco's modified Eagle's medium (DMEM, GIBCO) supplemented with $10 \%$ fetal bovine serum (FBS), $2 \mathrm{mM}$ glutamine, and Pen/Strep (100 U/ml, GIBCO) in an incubator with 5\% $\mathrm{CO}_{2}$ at $37^{\circ} \mathrm{C}$. MEFs at passage 2 were infected with retroviruses expressing Cre-PURO-IRES-GFP (pMSCVCre-PURO-IRES-GFP) or empty vector (pMSCV-PUROIRES-GFP). MEFs selected with puromycin $(2 \mu \mathrm{g} / \mathrm{ml})$ for 2 days were used for cell proliferation, transformation and invasion assays. The cell proliferation and transformation assay were performed as previously described [26]. For migration assay, MEFs were cultured with DMEM in $60 \mathrm{~mm}$ Petri dishes until confluent. Wound scratching was made using sterile $200 \mu \mathrm{l}$ tips followed with 2 washes in $1 \mathrm{x}$ PBS. After cultured continuously in fresh DMEM overnight, MEFs in dishes were fixed with $10 \%$ formalin for $15 \mathrm{~min}$ and photographed. Wounding healing (migration) rate was determined by a ratio of grids with cells at $16 \mathrm{hr}$ to grids at $0 \mathrm{hr}$.

\section{Bioluminescence imaging (BLI) in mice}

Bioluminescence imaging (BLI) was applied to noninvasively monitor the growth of prostate tumors of $\mathrm{Luc}^{p c^{+}}$; Pten $^{p c-1}$; Trp53 $3^{p c-1}$ male mice. Briefly, mice anesthetized with 3\% isoflurane were administered with D-Luciferin (Goldbio, MO) via I.P. at $125 \mathrm{mg} / \mathrm{kg}$ at $5 \mathrm{~min}$ before acquisition. Mice were then placed in the chamber of InVivo MS FX PRO optical imaging system (Carestream, $\mathrm{NY}$ ), and photons were collected for a period of $1 \mathrm{~min}$. The summary luminescent intensity of region of interest (ROI) was quantified using Molecular Imaging software v.5 -0.7.22 (Carestream, NY). To study CRPC in mice, mutant mice were subjected to castration surgery by removing their testicles at 14 weeks of age. The regressive and/or recurrent growth of prostate tumors in castrated mice was monitored weekly by BLI for firefly luciferase activities.

\section{Cell culture, transfection, shRNA and mutagenesis}

LNCaP, C4-2B (M.D. Anderson, TX), DU145, CWR22Rv1, PC3 (ATCC) human PCa cells were grown in RPMI 1640 medium with 10\% FBS and 1\% Pen/ Strep in an incubator with $5 \% \mathrm{CO}_{2}$ at $37^{\circ} \mathrm{C}$. $293 \mathrm{FT}$ cells were maintained in DMEM complemented with 10\% FBS and $1 \% \mathrm{Pen} / \mathrm{Strep}$ at $37^{\circ} \mathrm{C}$ with $5 \% \mathrm{CO}_{2}$. For transient transfection, cells at $70 \%$ confluence were transfected with plasmids using Lipofectamine 2000 (Invitrogen). Expression of target genes was determined 48-72 hr posttransfection using real-time qPCR or Western blotting as described [52]. To generate SKP2 shRNA plasmids, forward and reverse oligonucleotides (Supplementary Table S1) were suspended separately in $\mathrm{ddH}_{2} \mathrm{O}$ and then mixed in annealing buffer ( $5 \mu$ of 10 x NEB buffer 2 plus $35 \mu \mathrm{l}$ of $\mathrm{H}_{2} \mathrm{O}$ ). Annealed inserts were ligated into pLKO.1 TRC vector at AgeI and EcoRI sites. To knockdown SKP2 in cells, lentiviruses carrying SKP2 shRNA or scrambled sequence were prepared from 293FT cells transfected with a triple-plasmid system. Briefly, 293FT cells in $10 \mathrm{~cm}$ Lysine-coated petri dishes were co-transfected with $5 \mu \mathrm{g}$ of SKP2 scrambled or SKP2 shRNA plasmids, together with $3 \mu \mathrm{g}$ of psPAX 2 packaging plasmids, and 2 $\mu \mathrm{g}$ of pMD2.G envelope plasmids using Lipofectamine 2000. Forty eight hr post-transfection, viral supernatants were collected and filtered through $0.45 \mu \mathrm{m}$ filter. Fresh lentiviruses were applied to PC3 cells containing $5 \mu \mathrm{g}$ / $\mathrm{ml}$ of Polybrene. After additional $48 \mathrm{hr}$, infected cells were selected with $2 \mu \mathrm{g} / \mathrm{ml}$ of puromycin for 7 days and pooled for analysis [15]. Flag-JARID1B mutants were generated from pEV-Flag-JARID1B [53] using QuikChange II XL Site-Directed Mutagenesis Kit (Agilent Technologies, CA) according to manufacturer's instruction. The nucleotides sequences of various primers for SKP2 shRNA, and JARID1B mutants are shown in Supplementary Table S1.

\section{Real-time reverse transcription PCR}

Real-time reverse transcription PCR (real time RT-PCR) was performed according to the procedure as 
previously described [52]. Briefly, $5 \mu \mathrm{g}$ of total RNA extracted from PC3-scrambled or PC3-shSKP2 cells were subjected to cDNA synthesis by reverse transcription with SuperScript III first strand synthesis kit (Invitrogen). Realtime qPCR was performed with a Bio-Rad CFX96 Realtime system in triplicate using the forward and reverse primers listed in Supplementary Table S1.

\section{Western blotting and half-life determination of JARID1B}

Cell lysates were prepared in RIPA buffer $(1 \times \mathrm{PBS}$, $1 \%$ Nonidet P40/Triton X-100, 0.5\% sodium deoxycholate, $2 \mathrm{mM}$ EDTA, with or without $0.1 \%$ SDS) with protease inhibitor cocktail (Roche) followed with a brief sonication. Antibodies used were: rabbit anti-H3K4me3 (1:10,000, Cell Signaling, 9727), rabbit anti-H3 (1: 10,000, Abcam, Ab1791), rabbit anti-Skp2 (1:500, H-435, Santa Cruz, sc-7164), rabbit anti-JARID1B (1:10,000, Novus Biologicals, NB100-97821, NBP1-84352), mouse monoclonal anti- $\beta$-actin (1:10,000, Sigma, AC-74), mouse ant-Flag M2 affinity gel (Sigma, A2220), mouse anti-Flag M2 antibody (1:1000, Sigma, F1804), mouse anti-C-Myc $(1: 1000$, Santa Cruz, sc-40), mouse anti- $\beta$-Galactosidase (1:1000, LSBio, 10B2), mouse anti-TRAF6 (1:1000, Santa Cruz, D-10), rabbit anti-TRAF6 (1:1000, Cell Signaling, D21G3). The protein stability of JARID1B in PC3 cells were performed as previously described [26]. PC3-scrambled and PC3-shSKP2 cells were incubated with $100 \mu \mathrm{g} / \mathrm{ml}$ of cycloheximide (CHX, Sigma) in starvation medium to inhibit further protein synthesis. To determine the stability of JARID1B proteins, cell lysates were collected at indicated time points after CHX treatment, and then subjected to Western blotting analysis using rabbit anti-JARID1B antibodies. Protein bands were quantified with Image J software. The protein degradation rate is represented as half-life $\left(\mathrm{t}_{1 / 2}\right)$, which is defined as the time for $50 \%$ of the protein degraded.

\section{In vivo ubiquitination assay}

In vivo ubiquitination assays were performed as previously described [14, 54]. Briefly, HEK293T cells were transfected with Flag tagged JARID1B [53] or JARID1B mutants, HA tagged Ubiquitin WT or Ubiquitin mutants (K48-only and K63-only) [55], Myc tagged TRAF6 [56], along with or without Myc tagged SKP2 plasmids as indicated for $24 \mathrm{hr}$, then treated with $10 \mu \mathrm{M}$ of MG132 for additional $6 \mathrm{hr}$. Cells were lysed in 100 $\mu \mathrm{l}$ of SDS lysis buffer containing $50 \mathrm{mM}$ Tris- $\mathrm{HCl} \mathrm{pH}$ 7.5, $150 \mathrm{mM} \mathrm{NaCl}$ and 1\% SDS, and boiled for $10 \mathrm{~min}$ at $95^{\circ} \mathrm{C}$. Cell lysates were briefly sonicated, then diluted 10-fold with IP buffer (50 mM Tris- $\mathrm{HCl} \mathrm{pH} 7.5,150 \mathrm{mM}$ $\mathrm{NaCl}, 1 \%$ Triton X-100, 2 mM EDTA, protease inhibitor cocktail), and immunoprecipitated with $30 \mu$ l bed volume of anti-Flag M2 affinity gel at $4{ }^{\circ} \mathrm{C}$ overnight. After the beads were washed 5 times, the precipitated proteins were eluted with 2x SDS loading buffer, and applied to Western blotting analysis with indicated antibodies.

\section{Immunofluorescence (IF) and immunohistochemistry (IHC)}

Immunofluorescence (IF) stainings were performed as previously described [52]. Briefly, PC3-scrambled and PC3-shSKP2 cells were grown on cover slips in culture medium for $24 \mathrm{hr}$. Cells were fixed with methanol at $-20^{\circ} \mathrm{C}$, then incubated with indicated rabbit anti-JARID1B (1:250, Novus Biologicals, NB100-97821) and mouse anti-SKP2 (1:400, Invitrogen, 2C8D9), or mouse anti-CMyc (1:200, Santa Cruz, 9E10), or mouse anti-Fibrillarin (1:400, Abcam, ab4566), or mouse anti-Ub-K63 (1:200, Millipore, 05-1313), or mouse anti-TRAF6 (1:100, Santa Cruz, D-10), or mouse anti- $\beta$-Galactosidase (1:100, LSBio, 10B2). IHC staining on mouse tissue sections and human PCa tissue microarray were performed as previously reported [52]. Paraffin-embedded sections of mouse tissues in $5 \mu \mathrm{m}$ thickness were de-paraffinized in xylene for 3 X $10 \mathrm{~min}$, and rehydrated in graded alcohol, boiled in antigen retrieval citrate buffer, $\mathrm{pH} 6.0$ for 15 min, quenched in $3 \% \mathrm{H}_{2} \mathrm{O}_{2}$, and blocked with $10 \% \mathrm{FBS}$ in $1 \mathrm{x}$ PBS containing $0.1 \%$ Triton $\mathrm{X}-100$ and $1 \%$ BSA for $1 \mathrm{hr}$. These sections were then probed with primary antibodies: rabbit anti-Skp2 (1:50, Santa Cruz, H-435), rabbit anti-JARID1B (1:250, Novus Biologicals, NB10097821), rabbit anti-H3K4me3 (1:200, Cell Signaling, 9727), rabbit anti-TRAF6 (1:100, Cell signaling, D21G3), rabbit anti-Ki67 (Abcam 16667) for $16 \mathrm{hr}$. The sections were then stained with biotinylated secondary antibodies for $1 \mathrm{hr}$. The immune complex was visualized with $\mathrm{ABC}$ kit using the chromogen DAB substrate (Vector Labs). The nuclei were counterstained with Gill 3 Hematoxylin (Thermoscientific). Human prostate tissue microarray slides were purchased from Biomax, which consists of 35 cancer cases and 5 normal cases in 80 cores. Tissue sections were probed with primary antibodies: mouse antiSKP2 (1:250, Invitrogen, 2C8D9), rabbit anti-H3K4me3 (1:200, Cell Signaling, 9727). The scores of SKP2, H3K4me3 were graded as: 0 (negative staining), 1 (weak staining), 2 (moderate staining), or 3 (strong staining) according to their staining intensities [57].

\section{Statistical analysis}

Statistics analysis was performed using twotail Student's $t$-test. For correlation analysis, Pearson correlation test and Chi-square test were used. The values of $P<0.05$ were considered statistically significant.

\section{ACKNOWLEDGEMENTS}

We would like to thank Dr. Pier Paolo Pandolfi for his generous support with mouse models. FlagJARID1B plasmid is a kind gift from Dr. Ralf Janknecht. 
Myc-TRAF6 plasmid is a kind gift from Dr. Paul Bates. Ubiquitin and ubiquitin mutant plasmids are kindly provided by Dr. Edward W. Harhaj. We thank Dr. LaMonica V. Stewart for LNCaP cells, Dr. Renjie Jin for DU-145 and CWR22Rv1 cells. We thank Dr. J. Shawn Goodwin for his excellent assistance on microscopy. We gratefully thank Kieosha Williams of Human Tissue \& Pathology Core facility for the help on human tissue specimens. This work was supported in part by NIH grants MD004038, DK055748, U54 CA163069 and UL1 TR000445-06. Microscopy experiments and data analysis were performed through the use of Meharry Medical College Morphology Core supported in part by NIH grants U54 MD007593, G12 MD007586, R24 DA036420, and S10RR0254970.

\section{Conflicts of interest}

The authors have no potential conflicts of interest to disclose.

\section{REFERENCES}

1. Siegel R, Ma J, Zou Z, Jemal A. Cancer statistics. CA Cancer J Clin. 2014; 64:9-29.

2. Nakayama KI, Nakayama K. Ubiquitin ligases: cell-cycle control and cancer. Nat Rev Cancer. 2006; 6:369-381.

3. Carrano AC, Eytan E, Hershko A, Pagano M. SKP2 is required for ubiquitin-mediated degradation of the CDK inhibitor p27. Nat Cell Biol. 1999; 1:193-199.

4. Frescas D, Pagano M. Deregulated proteolysis by the F-box proteins SKP2 and beta-TrCP: tipping the scales of cancer. Nat Rev Cancer. 2008; 8:438-449.

5. Gstaiger M, Jordan R, Lim M, Catzavelos C, Mestan J, Slingerland J, Krek W. Skp2 is oncogenic and overexpressed in human cancers. Proc Natl Acad Sci U S A. 2001; 98:5043-5048.

6. Lin HK, Wang G, Chen Z, Teruya-Feldstein J, Liu Y, Chan $\mathrm{CH}$, Yang WL, Erdjument-Bromage H, Nakayama KI, Nimer S, Tempst P, Pandolfi PP. Phosphorylationdependent regulation of cytosolic localization and oncogenic function of Skp2 by Akt/PKB. Nat Cell Biol. 2009; 11:420-432.

7. Nakayama K, Nagahama H, Minamishima YA, Miyake S, Ishida N, Hatakeyama S, Kitagawa M, Iemura S, Natsume T, Nakayama KI. Skp2-mediated degradation of p27 regulates progression into mitosis. Dev Cell. :1-72. 2004; 6:661-672.

8. Lin HK, Chen Z, Wang G, Nardella C, Lee SW, Chan $\mathrm{CH}$, Yang WL, Wang J, Egia A, Nakayama KI, CordonCardo C, Teruya-Feldstein J, Pandolfi PP. Skp2 targeting suppresses tumorigenesis by Arf-p53-independent cellular senescence. Nature. 2010; 464:374-379.

9. Tian YF, Chen TJ, Lin CY, Chen LT, Lin LC, Hsing CH, Lee SW, Sheu MJ, Lee HH, Shiue YL, Huang HY, Pan
HY, Li CF, Chen SH. SKP2 overexpression is associated with a poor prognosis of rectal cancer treated with chemoradiotherapy and represents a therapeutic target with high potential. Tumour Biol. 2013; 34:1107-1117.

10. Chan CH, Lee SW, Li CF, Wang J, Yang WL, Wu CY, Wu J, Nakayama KI, Kang HY, Huang HY, Hung MC, Pandolfi PP, Lin HK. Deciphering the transcriptional complex critical for RhoA gene expression and cancer metastasis. Nat Cell Biol. 2010; 12:457-467.

11. Wang Z, Gao D, Fukushima H, Inuzuka H, Liu P, Wan L, Sarkar FH, Wei W. Skp2: a novel potential therapeutic target for prostate cancer. Biochim Biophys Acta. 2012; 1825:11-17.

12. Kim SY, Herbst A, Tworkowski KA, Salghetti SE, Tansey WP. Skp2 regulates Myc protein stability and activity. Mol Cell. 2003; 11:1177-1188.

13. von der Lehr N, Johansson S, Wu S, Bahram F, Castell A, Cetinkaya C, Hydbring P, Weidung I, Nakayama K, Nakayama KI, Soderberg O, Kerppola TK, Larsson LG. The F-box protein Skp2 participates in c-Myc proteosomal degradation and acts as a cofactor for c-Myc-regulated transcription. Mol Cell. 2003; 11:1189-1200.

14. Chan CH, Li CF, Yang WL, Gao Y, Lee SW, Feng Z, Huang HY, Tsai KK, Flores LG, Shao Y, Hazle JD, Yu D, Wei W, Sarbassov D, Hung MC, Nakayama KI, et al. The Skp2-SCF E3 ligase regulates Akt ubiquitination, glycolysis, herceptin sensitivity, and tumorigenesis. Cell. 2012; 149:1098-1111.

15. Li B, Lu W, Yang Q, Yu X, Matusik RJ, Chen Z. Skp2 regulates androgen receptor through ubiquitin-mediated degradation independent of Akt/mTOR pathways in prostate cancer. Prostate. 2014; 74:421-432.

16. Bianco-Miotto T, Chiam K, Buchanan G, Jindal S, Day TK, Thomas M, Pickering MA, O'Loughlin MA, Ryan NK, Raymond WA, Horvath LG, Kench JG, Stricker PD, Marshall VR, Sutherland RL, Henshall SM, et al. Global levels of specific histone modifications and an epigenetic gene signature predict prostate cancer progression and development. Cancer Epidemiol Biomarkers Prev. 2010; 19:2611-2622.

17. Ellinger J, Kahl P, von der Gathen J, Rogenhofer S, Heukamp LC, Gutgemann I, Walter B, Hofstadter F, Buttner R, Muller SC, Bastian PJ, von Ruecker A. Global levels of histone modifications predict prostate cancer recurrence. Prostate. 2010; 70:61-69.

18. Chi P, Allis CD, Wang GG. Covalent histone modifications- miswritten, misinterpreted and mis-erased in human cancers. Nat Rev Cancer. 2010; 10:457-469.

19. Ke XS, Qu Y, Rostad K, Li WC, Lin B, Halvorsen OJ, Haukaas SA, Jonassen I, Petersen K, Goldfinger N, Rotter V, Akslen LA, Oyan AM, Kall and KH. Genome-wide profiling of histone h3 lysine 4 and lysine 27 trimethylation reveals an epigenetic signature in prostate carcinogenesis. PLoS One. 2009; 4:e4687. 
20. Xiang Y, Zhu Z, Han G, Ye X, Xu B, Peng Z, Ma Y, Yu Y, Lin H, Chen AP, Chen CD. JARID1B is a histone H3 lysine 4 demethylase up-regulated in prostate cancer. Proc Natl Acad Sci U S A. 2007; 104:19226-19231.

21. Zhao JC, Yu J, Runkle C, Wu L, Hu M, Wu D, Liu JS, Wang Q, Qin ZS. Cooperation between Polycomb and androgen receptor during oncogenic transformation. Genome Res. 2012; 22:322-331.

22. Tsai YS, Lai CL, Lai CH, Chang KH, Wu K, Tseng SF, Fazli L, Gleave M, Xiao G, Gandee L, Sharifi N, Moro L, Tzai TS, Hsieh JT. The role of homeostatic regulation between tumor suppressor DAB2IP and oncogenic Skp2 in prostate cancer growth. Oncotarget. 2014; 5:6425-6436.

23. Liu H, Cheng EH, Hsieh JJ. Bimodal degradation of MLL by SCFSkp2 and APCCdc20 assures cell cycle execution: a critical regulatory circuit lost in leukemogenic MLL fusions. Genes Dev. 2007; 21:2385-2398.

24. Shen MM, Molecular genetics of prostate cancer: new prospects for old challenges. Genes Dev. 2010; 24:1967-2000.

25. Chen Z, Trotman LC, Shaffer D, Lin HK, Dotan ZA, Niki M, Koutcher JA, Scher HI, Ludwig T, Gerald W, CordonCardo C, Pandolfi PP. Crucial role of p53-dependent cellular senescence in suppression of Pten-deficient tumorigenesis. Nature. 2005; 436:725-730.

26. Chen Z, Carracedo A, Lin HK, Koutcher JA, Behrendt N, Egia A, Alimonti A, Carver BS, Gerald W, Teruya-Feldstein J, Loda M, Pandolfi PP. Differential p53-independent outcomes of p19(Arf) loss in oncogenesis. Sci Signal. 2009; 2:ra44.

27. Seligson DB, Horvath S, Shi T, Yu H, Tze S, Grunstein M, Kurdistani SK. Global histone modification patterns predict risk of prostate cancer recurrence. Nature. 2005; 435:1262-1266.

28. Yan YT, Stein SM, Ding J, Shen MM, Abate-Shen C. A novel PF/PN motif inhibits nuclear localization and DNA binding activity of the ESX1 homeoprotein. Mol Cell Biol. 2000; 20:661-671.

29. Safran M, Kim WY, Kung AL, Horner JW, DePinho RA, Kaelin WG Jr. Mouse reporter strain for noninvasive bioluminescent imaging of cells that have undergone Cremediated recombination. Mol Imaging. 2003; 2:297-302.

30. Lunardi A, Ala U, Epping MT, Salmena L, Clohessy JG, Webster KA, Wang G, Mazzucchelli R, Bianconi M, Stack EC, Lis R, Patnaik A, Cantley LC, Bubley G, Cordon-Cardo $\mathrm{C}$, Gerald WL, et al. A co-clinical approach identifies mechanisms and potential therapies for androgen deprivation resistance in prostate cancer. Nat Genet. 2013; 45:747-755.

31. Tsvetkov LM, Yeh KH, Lee SJ, Sun H, Zhang H. p27(Kip1) ubiquitination and degradation is regulated by the SCF(Skp2) complex through phosphorylated Thr187 in p27. Curr Biol. 1999; 9:661-664.
32. Wang G, Gao Y, Li L, Jin G, Cai Z, Chao JI, Lin HK. K63linked ubiquitination in kinase activation and cancer. Front Oncol. 2012; 2:5.

33. Radivojac P, Vacic V, Haynes C, Cocklin RR, Mohan A, Heyen JW, Goebl MG, Iakoucheva LM. Identification, analysis, and prediction of protein ubiquitination sites. Proteins. 2010; 78:365-380.

34. Sorrentino A, Thakur N, Grimsby S, Marcusson A, von Bulow V, Schuster N, Zhang S, Heldin CH, L and strom M. The type I TGF-beta receptor engages TRAF6 to activate TAK1 in a receptor kinase-independent manner. Nat Cell Biol. 2008; 10:1199-1207.

35. Thakur N, Sorrentino A, Heldin $\mathrm{CH}, \mathrm{L}$ and strom $\mathrm{M}$. TGF-beta uses the E3-ligase TRAF6 to turn on the kinase TAK1 to kill prostate cancer cells. Future Oncol. 2009; $5: 1-3$.

36. Tessarz P, Santos-Rosa H, Robson SC, Sylvestersen KB, Nelson CJ, Nielsen ML, Kouzarides T. Glutamine methylation in histone $\mathrm{H} 2 \mathrm{~A}$ is an RNA-polymerase-I-dedicated modification. Nature. 2014; 505:564-568.

37. Scott MS, Boisvert FM, McDowall MD, Lamond AI, Barton GJ. Characterization and prediction of protein nucleolar localization sequences. Nucleic Acids Res. 2010; 38:7388-7399.

38. Ohta K, Haraguchi N, Kano Y, Kagawa Y, Konno M, Nishikawa S, Hamabe A, Hasegawa S, Ogawa H, Fukusumi T, Uemura M, Nishimura J, Hata T, Takemasa I, Mizushima T, Noguchi Y, et al. Depletion of JARID1B induces cellular senescence in human colorectal cancer. Int J Oncol. 2013; 42:1212-1218.

39. Chicas A, Kapoor A, Wang X, Aksoy O, Evertts AG, Zhang MQ, Garcia BA, Bernstein E, Lowe SW. H3K4 demethylation by Jarid1a and Jarid1b contributes to retinoblastomamediated gene silencing during cellular senescence. Proc Natl Acad Sci U S A. 2012; 109:8971-8976.

40. Yang G, Ayala G, De Marzo A, Tian W, Frolov A, Wheeler TM, Thompson TC, Harper JW. Elevated Skp2 protein expression in human prostate cancer: association with loss of the cyclin-dependent kinase inhibitor p27 and PTEN and with reduced recurrence-free survival. Clin Cancer Res. 2002; 8:3419-3426.

41. Vogelstein B, Papadopoulos N, Velculescu VE, Zhou S, Diaz LA Jr. Kinzler KW. Cancer genome landscapes. Science. 2013; 339:1546-1558.

42. Blagosklonny MV. Cell cycle arrest is not yet senescence, which is not just cell cycle arrest: terminology for TOR-driven aging. Aging (Albany NY). 2012; 4:159-165.

43. Ellis L, Ku SY, Ramakrishnan S, Lasorsa E, Azabdaftari G, Godoy A, Pili R. Combinatorial antitumor effect of HDAC and the PI3K-Akt-mTOR pathway inhibition in a Pten defecient model of prostate cancer. Oncotarget. 2013; 4:2225-2236. 
44. Gibbons RJ. Histone modifying and chromatin remodelling enzymes in cancer and dysplastic syndromes. Hum Mol Genet. 2005; 14:R85-92.

45. Waldmann T, Schneider R. Targeting histone modifications-epigenetics in cancer. Curr Opin Cell Biol. 2013; 25:184-189.

46. Zhang K, Dent SY. Histone modifying enzymes and cancer: going beyond histones. J Cell Biochem. 2005; 96:1137-1148.

47. Chinaranagari S, Sharma P, Chaudhary J. EZH2 dependent $\mathrm{H} 3 \mathrm{~K} 27 \mathrm{me} 3$ is involved in epigenetic silencing of ID4 in prostate cancer. Oncotarget. 2014; 5:7172-7182.

48. Bueno MT, Richard S. SUMOylation negatively modulates target gene occupancy of the KDM5B, a histone lysine demethylase. Epigenetics. 2013; 8:1162-1175.

49. Yamane K, Tateishi K, Klose RJ, Fang J, Fabrizio LA, Erdjument-Bromage H, Taylor-Papadimitriou J, Tempst P, Zhang Y. PLU-1 is an H3K4 demethylase involved in transcriptional repression and breast cancer cell proliferation. Mol Cell. 2007; 25:801-812.

50. Roesch A, Vultur A, Bogeski I, Wang H, Zimmermann KM, Speicher D, Korbel C, Laschke MW, Gimotty PA, Philipp SE, Krause E, Patzold S, Villanueva J, Krepler C, Fukunaga-Kalabis M, Hoth M, et al. Overcoming intrinsic multidrug resistance in melanoma by blocking the mitochondrial respiratory chain of slow-cycling JARID1B(high) cells. Cancer Cell. 2013; 23:811-825.
51. Pickart CM, Eddins MJ. Ubiquitin: structures, functions, mechanisms. Biochim Biophys Acta. 2004; 1695:55-72.

52. Lu W, Xie Y, Ma Y, Matusik RJ, Chen Z. ARF represses androgen receptor transactivation in prostate cancer. Mol Endocrinol. 2013; 27:635-648.

53. Kim J, Shin S, Subramaniam M, Bruinsma E, Kim TD, Hawse JR, Spelsberg TC, Janknecht R. Histone demethylase JARID1B/KDM5B is a corepressor of TIEG1/KLF10. Biochem Biophys Res Commun. 2010; 401:412-416.

54. Qi J, Tripathi M, Mishra R, Sahgal N, Fazli L, Ettinger S, Placzek WJ, Claps G, Chung LW, Bowtell D, Gleave M, Bhowmick N, Ronai ZA. The E3 ubiquitin ligase Siah2 contributes to castration-resistant prostate cancer by regulation of androgen receptor transcriptional activity. Cancer Cell. 2013; 23:332-346.

55. Shembade N, Ma A, Harhaj EW. Inhibition of NF-kappaB signaling by A20 through disruption of ubiquitin enzyme complexes. Science. 2010; 327:1135-1139.

56. Cocka LJ, Bates P. Identification of alternatively translated Tetherin isoforms with differing antiviral and signaling activities. PLoS Pathog. 2012; 8:e1002931.

57. Lakshmikanthan V, Zou L, Kim J, Michal A, Nie Z, Messias NC, Benovic JL, Daaka Y. Identification of betaArrestin2 as a corepressor of androgen receptor signaling in prostate cancer. Proc Natl Acad Sci U S A. 2009; 106:9379-9384. 